\title{
A RIGOROUS FORMULATION OF THE COSMOLOGICAL NEWTONIAN LIMIT WITHOUT AVERAGING
}

\author{
TODD A. OLIYNYK
}

\begin{abstract}
We prove the existence of a large class of one-parameter families of cosmological solutions to the Einstein-Euler equations that have a Newtonian limit. This class includes solutions that represent a finite, but otherwise arbitrary, number of compact fluid bodies. These solutions provide exact cosmological models that admit Newtonian limits but, are not, either implicitly or explicitly, averaged.
\end{abstract}

\section{INTRODUCTION}

Gravitating perfect fluids are governed by the Einstein-Euler equations

$$
G^{i j}=\frac{8 \pi G}{c^{4}} T^{i j} \quad \text { and } \quad \nabla_{i} T^{i j}=0,
$$

where

$$
T^{i j}=\left(\rho+c^{-2} p\right) v^{i} v^{j}+p g^{i j},
$$

with $\rho$ the fluid density, $p$ the fluid pressure, $v^{i}$ the fluid four-velocity normalized by $v^{i} v_{i}=-c^{2}, c$ the speed of light, and $G$ the Newtonian gravitational constant. By suitably rescaling (see [8]), these equations can be written as

$$
G^{i j}=2 \epsilon^{4} T^{i j} \quad \text { and } \quad \nabla_{i} T^{i j}=0
$$

where

$$
T^{i j}=\left(\rho+\epsilon^{2} p\right) v^{i} v^{j}+p g^{i j} \quad \text { and } \quad v^{i} v_{i}=-\frac{1}{\epsilon^{2}} .
$$

In the article 10, we proved the existence of a large class of one-parameter families of solutions to this system defined for $0<\epsilon<\epsilon_{0}$ that

(i) exist on a common piece of spacetime of the form $M=[0, T) \times \mathbb{T}^{3}$,

(ii) converge as $\epsilon \searrow 0$ to solutions of the cosmological Poisson-Euler equations of Newtonian gravity, and

(iii) are differentiable in $\epsilon$ to any prescribed order $\ell \in \mathbb{N}$.

The properties (i)-(iii) guarantee that these one parameter families of solutions to the Einstein-Euler equations have valid Newtonian limits and admit post-Newtonian expansions to order $\ell / 2$. However, in order to establish the existence of these solutions, we required that the two following conditions are satisfied by the initial fluid density $\left.\rho\right|_{t=0}=\rho_{0}$ and fluid three-velocity $\left.v^{I}\right|_{t=0}=w_{0}^{I}$ :

$$
\rho_{0}(x)>0 \quad \forall x \in \mathbb{T}^{3},
$$

and

$$
\int_{\mathbb{T}^{3}} \rho_{0} w_{0}^{I} d^{3} x=0 .
$$

Here, we are using $x=\left(x^{I}\right)(I=1,2,3)$ to denote the standard periodic coordinates with period 1 on the torus $\mathbb{T}^{3}=S^{1} \times S^{1} \times S^{1}$, and $t=x^{0}$ to denote an absolute Newtonian time coordinate on the interval $[0, T)$.

2000 Mathematics Subject Classification. 83C25. 
The main aim of this article is to remove the conditions (1.2)-(1.3) on the initial data. Although, we do not discuss the existence of post-Newtonian expansions in this article, it is not difficult to see that the results of this article can be combined with those of [10] to prove the existence of post-Newtonian expansions to arbitrary order that do not satisfy (1.2)-(1.3).

From our point of view, there are two important reasons for removing the conditions (1.2)-(1.3). This first reason is that (1.3) is an exact averaging condition that cannot be expected to be exactly satisfied for real systems. The second is that by removing (1.2), we can choose an initial fluid density of the form

$$
\rho_{0}=\sum_{\nu=0}^{N} \rho_{0, \nu}
$$

where $N$ is any integer, and

$$
\operatorname{dist}\left(\operatorname{supp} \rho_{0, \nu}, \operatorname{supp} \rho_{0, \mu}\right)>0 \quad \nu \neq \mu .
$$

This initial data represents a finite but otherwise arbitrary number of compact fluid bodies (i.e. stars). Thus, solutions generated by this initial data can be used to model an arbitrary collection of stars, and therefore provide an exact model for the universe. Consequently, we obtain one-parameter families of cosmological solutions that admit Newtonian limits, and are not, either implicitly or explicitly, averaged.

To formulate the Newtonian limit, we require (see [10]) the following FLRW dust solution $\left(g_{i j}^{\epsilon}=\right.$ $\left.h_{i j}^{\epsilon}, v_{\epsilon}^{i}=\xi^{i}, \rho_{\epsilon}=\mu_{\epsilon}, p_{\epsilon}=0\right)$ of (1.1):

$$
\begin{aligned}
h_{i j}^{\epsilon} & =-\frac{1}{\epsilon^{2}} \delta_{i}^{0} \delta_{j}^{0}+a_{\epsilon} \delta_{i}^{I} \delta_{j}^{J} \delta_{I J}, \\
\xi^{i} & =\delta_{0}^{i}, \\
\mu_{\epsilon} & =\frac{3}{8}\left[\frac{a_{\epsilon}^{\prime}}{a_{\epsilon}}\right]^{2},
\end{aligned}
$$

where $a_{\epsilon}=a_{\epsilon}(t)$ and $\mu_{\epsilon}=\mu_{\epsilon}(t)$ satisfy the differential equations

$$
\frac{a_{\epsilon}^{\prime \prime}}{a_{\epsilon}}-\frac{1}{2}\left[\frac{a_{\epsilon}^{\prime}}{a_{\epsilon}}\right]^{2}+\frac{2}{3} \mu_{\epsilon}=0
$$

and

$$
\mu_{\epsilon}^{\prime}+\frac{3}{2} \frac{a_{\epsilon}^{\prime}}{a_{\epsilon}} \mu_{\epsilon}=0,
$$

respectively. Here, we are using

$$
(\cdot)^{\prime}=\frac{d}{d t}(\cdot) .
$$

The differential equations (1.7)-(1.8) can be integrated explicitly to give

$$
a_{\epsilon}(t)=a_{\epsilon}(0)\left(\sqrt{\frac{3 \sigma(\epsilon)}{2}} t+1\right)^{\frac{4}{3}},
$$

and

$$
\mu_{\epsilon}(t)=\frac{2}{3}\left(t+\sqrt{\frac{2}{3 \sigma(\epsilon)}}\right)^{-2},
$$

where $a_{\epsilon}(0)$ and $\sigma(\epsilon)=\mu_{\epsilon}(0)$ are arbitrary (positive) functions that are analytic in a neighborhood of $\epsilon=0$. We fix the arbitrary length scale by setting $a_{\epsilon}(0)=1$, while $\sigma(\epsilon)$ will be determined by later considerations. Also, to reduce notation we will drop the $\epsilon$ from the function $a$ and $\mu$ except in situations where we want to emphasize the $\epsilon$-dependence. 
In this article, we use a slight variation of the approach used in [10] to analyze the limit $\epsilon \searrow 0$. The first step in the analysis is to replace the metric $g_{i j}$ and the fluid velocity $v^{i}$ with variables that are compatible with the limit $\epsilon \searrow 0$. The new gravitational variable $\bar{u}^{i j}$ is defined by

$$
g^{i j}=\frac{q^{i j}}{\sqrt{-|h| \operatorname{det} q^{k l}}} \quad\left(|h|=-\operatorname{det}\left(h_{i j}\right)\right)
$$

where

$$
q^{i j}=h^{i j}+\epsilon^{2} J_{k}^{i} J_{l}^{j} \bar{u}^{k l} \text { and } \quad J_{i}^{j}=\epsilon \delta_{0}^{j} \delta_{i}^{0}+\delta_{I}^{j} \delta_{i}^{I},
$$

while the new fluid four-velocity $w^{i}$ is defined by

$$
v^{i}=\left(1+\epsilon w^{0}\right) \delta_{0}^{i}+\delta_{I}^{i} w^{I} .
$$

As in 8,9], we use Makino's technique [7,11 to generate perfect fluid solutions with compact support. This requires the use of an isentropic equation of state of the form

$$
p=K \rho^{(n+1) / n},
$$

where $K \in \mathbb{R}_{>0}, n \in \mathbb{N}$. This allows for the introduction of the density variable

$$
\rho=\frac{1}{(4 K n(n+1))^{n}} \alpha^{2 n}
$$

which is used to formulate the Euler equations as a symmetric hyperbolic system that is regular across the fluid-vacuum interface. In this way, it is possible to construct solutions to the Einstein-Euler equations that represent compact gravitating fluid bodies (i.e. stars) both in the Newtonian and relativistic setting [7,11]. Although, these solutions do not include static stars of finite radius [11, they are general enough to understand the mathematical issues involved in the Newtonian limit.

The main result of this article is to show that solutions to the following equations, which we refer to as the cosmological Poisson-Euler-Makino equations, rigorously approximate fully relativistic solutions to the Einstein-Euler equations (1.1) up to an error term of order $\epsilon$ as measured in suitable Sobolev spaces:

$$
\begin{aligned}
\partial_{t} \tilde{\alpha} & =-\tilde{w}^{I} \partial_{I} \tilde{\alpha}-\frac{\tilde{\alpha}}{2 n} \partial_{I} \tilde{w}^{I}-\frac{3 \tilde{a}^{\prime}}{4 n \tilde{a}} \tilde{\alpha}, \\
\partial_{t} \tilde{w}^{J} & =-\tilde{w}^{I} \partial_{I} \tilde{w}^{J}-\frac{\tilde{\alpha}}{2 n \tilde{a}} \partial^{J} \tilde{\alpha}-\frac{\tilde{a}^{\prime}}{\tilde{a}} \tilde{w}^{J}+g^{J}, \\
\Delta \tilde{\Phi} & =4 \tilde{a}(\tilde{\rho}-\tilde{\mu}) \quad\left(\tilde{\rho}=(4 K n(n+1))^{-n} \tilde{\alpha}^{2 n}\right),
\end{aligned}
$$

where

$$
\begin{gathered}
\tilde{a}=a_{0}, \quad \tilde{\mu}=\mu_{0}, \\
g^{J}=-\frac{1}{\tilde{a}}\left(\frac{3}{2} \frac{\tilde{a}^{\prime}}{\tilde{a}} \frac{1}{\tilde{\mu}} \int_{\mathbb{T}^{3}} \tilde{\rho} \tilde{w}^{J} d^{3} x+\frac{1}{4} \partial^{J} \tilde{\Phi}\right),
\end{gathered}
$$

and $\langle\cdot \mid \cdot\rangle_{L^{2}}$ is the standard $L^{2}$ inner-product on $\mathbb{T}^{3}$, i.e.

$$
\left\langle\psi_{1} \mid \psi_{2}\right\rangle_{L^{2}}=\int_{[0,1]^{3}} \psi_{1}(x) \psi_{2}(x) d^{3} x
$$

Here, and for the rest of the article,

$$
\Delta=\delta^{I J} \partial_{I} \partial_{J}
$$

will denote the flat Laplacian, and we set

$$
\partial^{J}=\delta^{I J} \partial_{I} .
$$


In terms of the fluid density $\tilde{\rho}$, equations (1.16)-(1.18) take the form

$$
\begin{aligned}
\partial_{t} \tilde{\rho} & =-\tilde{w}^{I} \partial_{I} \tilde{\rho}-\tilde{\rho} \partial_{I} \tilde{w}^{I}-\frac{3}{2} \frac{\tilde{a}^{\prime}}{\tilde{a}} \tilde{\rho}, \\
\partial_{t} \tilde{w}^{J} & =-\tilde{w}^{I} \partial_{I} \tilde{w}^{J}-\frac{1}{\tilde{a} \tilde{\rho}} \partial^{J} p(\tilde{\rho})-\frac{\tilde{a}^{\prime}}{\tilde{a}} \tilde{w}^{J}+g^{J}, \\
\Delta \tilde{\Phi} & =4 \tilde{a}(\tilde{\rho}-\tilde{\mu}),
\end{aligned}
$$

which we refer to as the cosmological Poisson-Euler equations. We note that these equations agree with the Newton-Cartan field equations for a gravitating fluid formulated in adapted coordinates [5, 12.

An important point is that the constant $\sigma(0)=\tilde{\mu}(0)$ is not arbitrary but is determined by the initial density

$$
\tilde{\rho}_{0}=\left.\tilde{\rho}\right|_{t=0}
$$

according to

$$
\sigma_{0}:=\tilde{\mu}(0)=\int_{\mathbb{T}^{3}} \tilde{\rho}_{0} d^{3} x>0 .
$$

From equations (1.8) and (1.20), it is not difficult to see that this average is preserved under evolution

$$
\tilde{\mu}(t)=\int_{\mathbb{T}^{3}} \tilde{\rho}(t) d^{3} x,
$$

which in turn implies (see (1.6) ) that

$$
\tilde{a}(t)=\exp \left(\int_{0}^{t}\left(\frac{8}{3} \int_{\mathbb{T}^{3}} \tilde{\rho}(s) d^{3} x\right)^{\frac{1}{2}} d s\right) .
$$

Also, setting

$$
\zeta^{J}=\int_{\mathbb{T}^{3}} \tilde{\rho} \tilde{w}^{J} d^{3} x
$$

it follows directly from the cosmological Poisson-Euler equations (1.20)-(1.22) that $\zeta^{J}$ satisfies the equation

which can be integrated to give

$$
\zeta^{J^{\prime}}=-4 \frac{\tilde{a}^{\prime}}{\tilde{a}} \zeta^{J}
$$

$$
\zeta^{J}(t)=\frac{1}{\tilde{a}(t)^{4}} \int_{\mathbb{T}^{3}} \tilde{\rho}_{0} \tilde{w}_{0}^{J} d^{3} x
$$

where

$$
\tilde{w}_{0}^{J}=\left.\tilde{w}^{J}\right|_{t=0}
$$

is the initial fluid 3-velocity. Using (1.27), the acceleration due to gravity (1.19) can be reexpressed in the form

$$
g^{J}=-\frac{1}{\tilde{a}}\left(\frac{4}{\tilde{a}^{\prime} \tilde{a}^{3}} \int_{\mathbb{T}^{3}} \tilde{\rho}_{0} \tilde{w}_{0}^{J} d^{3} x+\frac{1}{4} \partial^{J} \tilde{\Phi}\right) .
$$

For purposes of interpretation, it is often useful to introduce Galilei coordinates [5, 12, These coordinates are defined as follows: suppose $\left\{\tilde{\rho}(t, x), w^{I}(t, x), \Phi(t, x)\right\}$ is a solution of the cosmological Poisson-Euler equations (1.20)-(1.22) on $M=[0, T) \times \mathbb{T}^{3}$. Then, letting $\tilde{M}=[0, T) \times \mathbb{R}^{3}$ denote the covering space, we define a diffeomorphism on $\tilde{M}$ by

$$
\psi: \tilde{M} \longrightarrow \tilde{M}:(t, x) \longmapsto(t, x / \sqrt{\tilde{a}(t)}) .
$$


Lifting the cosmological Poisson-Euler equations to $\tilde{M}$, and then pulling back by $\psi$ shows that 1

$$
\begin{aligned}
\hat{\rho}(t, x) & =\tilde{\rho}(t, x / \sqrt{\tilde{a}(t)}), \\
\hat{w}^{J}(t, x) & =\sqrt{\tilde{a}(t)} \hat{w}^{J}(t, x / \sqrt{\tilde{a}(t)})+\frac{1}{2} \frac{\tilde{a}^{\prime}(t)}{\tilde{a}} x^{J}, \\
\hat{\Phi}(t, x) & =\tilde{\Phi}(t, x / \sqrt{\tilde{a}(t)}),
\end{aligned}
$$

satisfy

$$
\begin{aligned}
\partial_{t} \hat{\rho} & =-\hat{w}^{I} \partial_{I} \hat{\rho}-\hat{\rho} \partial_{I} \hat{w}^{I}, \\
\partial_{t} \hat{w}^{J} & =-\hat{w}^{I} \partial_{I} \hat{w}^{J}-\frac{1}{\hat{\rho}} \partial^{J} p(\hat{\rho})+\hat{g}^{J}, \\
\Delta \hat{\Phi} & =4(\hat{\rho}-\tilde{\mu}),
\end{aligned}
$$

where

$$
\hat{g}^{J}=-\frac{4}{\tilde{a}^{\prime} \tilde{a}^{7 / 2}} \int_{\mathbb{T}^{3}} \tilde{\rho}_{0} \tilde{w}_{0}^{J} d^{3} x-\frac{1}{4} \partial^{J} \hat{\Phi}-\frac{\tilde{\mu}}{3} x^{J} .
$$

A Newtonian potential can be defined by

$$
\check{\Phi}=\frac{\hat{\Phi}}{4}+\frac{\tilde{\mu}}{6} \delta_{I J} x^{I} x^{J}+\frac{4}{\tilde{a}^{\prime} \tilde{a}^{7 / 2}} \delta_{I J} x^{I} \int_{\mathbb{T}^{3}} \tilde{\rho}_{0} \tilde{w}_{0}^{J} d^{3} x .
$$

This potential satisfies the Poisson equation

$$
\Delta \check{\Phi}=\hat{\rho},
$$

while the acceleration due to gravity $\hat{g}^{J}$ takes the familiar form

$$
\hat{g}^{J}=-\partial^{J} \check{\Phi} \text {. }
$$

Together, equations (1.31), (1.32), (1.36), and (1.37) show that solutions to the cosmological PoissonEuler equations determine solutions to the standard Poisson-Euler equations on the covering space $\tilde{M}$.

1.1. Notation. Before proceeding, we first fix our notation and introduce a number of function spaces that will be used in this article. Given a finite dimensional vector space $V$, we let $H^{s}(V)$ denote the standard Sobolev space of $V$-valued maps on $\mathbb{T}^{3}$. When $V=\mathbb{R}$, we just write $H^{s}$. The only two vector spaces that will be used in this article are $\mathbb{R}^{N}$ and the space of symmetric matrices $\mathbb{S}_{N}=\left\{\left(u^{i j}\right) \in\right.$ $\left.\mathbb{M}_{N \times N} \mid u^{i j}=u^{j i}\right\}$.

We denote the projection operator onto the $L^{2}$ orthogonal complement of the constant function 1 by

$$
\Pi(\psi)=\psi-\langle 1 \mid \psi\rangle_{L^{2}} 1 \quad \forall \psi \in L^{2}\left(\mathbb{T}^{3}\right) .
$$

Given $\left\{\mathbf{e}_{\alpha}\right\}_{\alpha=1}^{N}$ any basis for $V$, we use this projection to define

$$
\bar{H}^{s}(V)=\left\{\psi=\sum_{\alpha=1}^{N} \psi^{\alpha} \mathbf{e}_{\alpha} \in H^{s}(V) \mid\left\langle 1 \mid \psi^{\alpha}\right\rangle_{L^{2}}=0\right\} .
$$

We also define the standard hyperbolic evolution spaces

$$
X_{T, \ell, s}(V)=\bigcap_{p=0}^{\ell+1} C^{p}\left([0, T), H^{s-p}(V)\right) .
$$

and write $X_{T, \ell, s}$ if $V=\mathbb{R}$.

\footnotetext{
${ }^{1}$ In the Newton-Cartan theory, the fluid velocity 3 -vector $\tilde{w}^{I}$ is the spatial part of a 4 -vector $\tilde{w}=\partial_{t}+\tilde{w}^{I} \partial_{I}$ [12. The formula (1.29) follows from the calculating the spatial components of $\hat{w}=\psi^{*} \tilde{w}$. The other two formulas (1.28) and (1.30) follow from the definition of the pullback, i.e. $\hat{\rho}=\psi^{*} \tilde{\rho}$ and $\hat{\Phi}=\psi^{*} \tilde{\Phi}$.
} 
1.2. Main result. The main results of this article is the following Theorem which establishes the existence of a wide class of 1-parameter families of solutions to the Einstein-Euler equations that converge in the limit $\epsilon \searrow 0$ to solutions of the cosmological Poisson-Euler equations (1.20)-(1.22). See Section 5 for the proof.

Theorem 1.1. Suppose $K>0, n, s, \ell \in \mathbb{Z}_{\geq 0}, s \geq 3+\ell, \tilde{\alpha}_{0} \in H^{s}, \tilde{w}_{0}^{I} \in H^{s}\left(\mathbb{R}^{3}\right), \breve{u}_{0}^{I J} \in \bar{H}^{s}\left(\mathbb{S}_{3}\right)$ , $\breve{u}^{I J} \in \bar{H}^{s+1}\left(\mathbb{S}_{3}\right), T_{0}$ as defined in Proposition 4.1, and $\sigma_{0}>0$ is defined by (1.23) where $\tilde{\rho}_{0}=$ $(4 K n(n+1))^{-n} \tilde{\alpha}_{0}^{2 n}$. Then for $\epsilon_{0}$ small enough, there exists a $T \in\left(0, T_{0}\right)$ independent of $\epsilon \in\left(0, \epsilon_{0}\right)$, and maps

$$
\begin{gathered}
\bar{u}_{\epsilon}^{i j} \in X_{T, \ell+1, s+1}\left(\mathbb{S}_{4}\right) \quad 0<\epsilon<\epsilon_{0}, \\
\alpha_{\epsilon} \in X_{T, \ell, s}, \quad w_{\epsilon}^{i}(t) \in X_{T, \ell, s}\left(\mathbb{R}^{4}\right) \quad 0<\epsilon<\epsilon_{0}, \\
\tilde{\alpha} \in X_{T_{0}, \ell, s}, \quad \tilde{w}^{I} \in X_{T_{0}, \ell, s}\left(\mathbb{R}^{3}\right), \quad \tilde{\Phi} \in X_{T_{0}, \ell+2, s+2}, \\
\sigma \in C^{\omega}\left(\left(-\epsilon_{0}, \epsilon_{0}\right), \mathbb{R}_{>\sigma_{0} / 2}\right), \quad \sigma(0)=\sigma_{0}, \\
y^{J} \in C^{\omega}\left(\left(-\epsilon_{0}, \epsilon_{0}\right), \mathbb{R}^{3}\right), \quad y^{J}(0)=-\frac{1}{\sigma_{0}} \int_{\mathbb{T}^{3}} \tilde{\rho}_{0} \tilde{w}_{0}^{J} d^{3} x
\end{gathered}
$$

such that

(i)

$$
\begin{aligned}
\left.\alpha_{\epsilon}\right|_{t=0} & =\tilde{\alpha}_{0}, \\
\left.\left(w_{\epsilon}^{j}\right)\right|_{t=0} & =\left(w_{\epsilon}^{0}, \tilde{w}_{0}^{I}\right), \\
\left.\left(\bar{u}_{\epsilon}^{i j}\right)\right|_{t=0} & =\left(\begin{array}{cc}
\breve{u}_{\epsilon}^{00} & \frac{1}{\epsilon} y^{J}(\epsilon)+\bar{u}_{\epsilon}^{0 J} \\
\frac{1}{\epsilon} y^{J}(\epsilon)+\breve{u}_{\epsilon}^{J 0} & \epsilon \breve{u}^{I J}
\end{array}\right), \\
\left.\left(\partial_{t} \bar{u}_{\epsilon}^{i j}\right)\right|_{t=0} & =\left(\begin{array}{cc}
\left.\partial_{t} \bar{u}_{\epsilon}^{00}\right|_{t=0} & \left.\partial_{t} \bar{u}_{\epsilon}^{0 J}\right|_{t=0} \\
\left.\partial_{t} \bar{u}_{\epsilon}^{0 J}\right|_{t=0} & \breve{u}_{0}^{I J}
\end{array}\right)
\end{aligned}
$$

where

$$
\begin{aligned}
\left.\partial_{t} \bar{u}_{\epsilon}^{00}\right|_{t=0} & =-\frac{1}{\epsilon} \partial_{I} \breve{u}_{\epsilon}^{I 0}-\frac{3}{2} \ln (a)^{\prime} \breve{u}_{\epsilon}^{00}-\frac{1}{2} a^{\prime} \delta_{I J} \epsilon \breve{u}^{I J}, \\
\left.\partial_{t} \bar{u}_{\epsilon}^{0 J}\right|_{t=0} & =-\partial_{I} \breve{u}^{I J}-\frac{5}{2} \ln (a)^{\prime}\left(\frac{1}{\epsilon} y^{J}(\epsilon)+\breve{u}_{\epsilon}^{0 J}\right),
\end{aligned}
$$

(ii) $\left\{\bar{u}_{\epsilon}^{i j}(t, x), \alpha_{\epsilon}(t, x), w_{\epsilon}^{i}(t, x)\right\}$ determines, via the formulas (1.11), (1.13), and (1.15), a 1-parameter family of unique solutions to the Einstein-Euler equations (1.1) in the harmonic gauge on the common spacetime region $(t, x) \in M=[0, T) \times \mathbb{T}^{3}$,

(iii) $\left\{\tilde{\Phi}(t, x), \tilde{\rho}(t, x), \tilde{w}^{I}(t, x)\right\}$ solves the cosmological Euler-Poisson equations (1.20)-(1.22) on the spacetime region $M$, and

(iv) there exists a constant $C>0$ independent of $\epsilon \in\left(0, \epsilon_{0}\right)$ such that

$$
\begin{gathered}
\epsilon^{-1}\left\|v_{\epsilon}^{0}(t)-1\right\|_{H^{s-1}}+\left\|v_{\epsilon}^{I}(t)-\tilde{w}^{I}(t)\right\|_{H^{s-1}}+\left\|\rho_{\epsilon}(t)-\tilde{\rho}(t)\right\|_{H^{s-1}} \leq C \epsilon, \\
\left\|\epsilon \bar{u}_{\epsilon}^{i j}(t)-\tilde{u}^{i j}(t)\right\|_{H^{s-1}}+\left\|\partial_{K} \bar{u}_{\epsilon}^{i j}(t)-\delta_{0}^{i} \delta_{0}^{j} \tilde{\Phi}(t)\right\|_{H^{s-1}} \leq C \epsilon,
\end{gathered}
$$

and

$$
\begin{aligned}
\left\|\epsilon \partial_{t} \bar{u}_{\epsilon}^{00}(t)\right\|_{H^{s-1}} & \leq C \epsilon, \\
\left\|\epsilon \partial_{t} \bar{u}_{\epsilon}^{0 J}(t)-\tilde{u}_{0}^{0 J}(t)+\frac{a^{\prime}(t)}{2 a(t)} \tilde{u}^{0 J}(t)\right\|_{H^{s-1}} & \leq C \epsilon, \\
\left\|\epsilon \partial_{t} \bar{u}_{\epsilon}^{I J}(t)-\tilde{u}_{0}^{I J}(t)\right\|_{H^{s-1}} & \leq C \epsilon,
\end{aligned}
$$


for all $(t, \epsilon) \in[0, T) \times\left(0, \epsilon_{0}\right)$, where

$$
\begin{aligned}
& \tilde{u}_{0}^{i j}=2 \delta_{0}^{(i} \delta_{J}^{j)}\left(-\left(\frac{1}{\tilde{\mu}} \zeta^{J}\right)^{\prime}-\frac{\tilde{a}^{\prime}}{2 \tilde{a}} \frac{1}{\tilde{\mu}} \zeta^{J}\right), \\
& \tilde{u}^{i j}=2 \delta_{0}^{(i} \delta_{J}^{j)}\left(-\frac{2}{\tilde{\mu}} \zeta^{J}\right),
\end{aligned}
$$

and $\tilde{\mu}, \tilde{a}$, and $\zeta^{J}$ are defined by equations (1.24), (1.25), and (1.26).

\section{The Einstein-Euler equations}

In this section, we adapt the formulation used in [10] to write the Einstein-Euler equations in a form suitable to analyze the limit $\epsilon \searrow 0$ for initial data that does not satisfy (1.2)-(1.3). The main change from the formalism used in [10] is that scale factor $a_{\epsilon}(t)$ appearing in the FLRW metric is now $\epsilon$-dependent through the function $\sigma(\epsilon)$. This additional freedom allows us to construct initial data that is more general than that in [10].

2.1. Reduced Einstein Equations. To derive a suitable symmetric hyperbolic system for the gravitational field equations, we introduce new coordinates related to old ones by the rescaling

$$
\bar{x}^{0}=x^{0} / \epsilon, \quad \bar{x}^{J}=x^{J},
$$

and let

$$
\bar{\partial}_{i}=\frac{\partial}{\partial \bar{x}^{i}} .
$$

In the new coordinates, the spacetime metric $\bar{g}_{i j}$ and the FLRW metric $\bar{h}_{i j}$ (see (1.4)) are given by

$$
\bar{g}_{i j}=J_{i}^{k} J_{j}^{k} g_{i j} \quad \text { and } \quad \bar{h}_{i j}=-\delta_{i}^{0} \delta_{j}^{0}+a \delta_{i}^{I} \delta_{i}^{J} \delta_{I J}
$$

respectively, where $J_{i}^{j}$ is defined in (1.12). The non-zero independent components of the Christoffel symbols $\bar{\gamma}_{i j}^{k}$ and the curvature $\overline{\mathcal{R}}_{i j k l}$ of the metric $\bar{h}_{i j}$ are:

$$
\begin{aligned}
\bar{\gamma}_{0 I}^{I} & =\frac{\epsilon}{2} \frac{a^{\prime}}{a}, \\
\bar{\gamma}_{I I}^{0} & =\frac{\epsilon}{2} a^{\prime}, \\
\overline{\mathcal{R}}_{0 I 0 I} & =-\frac{\epsilon^{2}}{4} \frac{2 a a^{\prime \prime}-\left(a^{\prime}\right)^{2}}{a},
\end{aligned}
$$

and

$$
\overline{\mathcal{R}}_{2121}=\overline{\mathcal{R}}_{1313}=\overline{\mathcal{R}}_{2323}=\frac{\epsilon^{2}}{4}\left(a^{\prime}\right)^{2} .
$$

As discussed in the introduction, we take the symmetric 2-tensor $\bar{u}^{i j}$ as our primary gravitational variable where

$$
\bar{u}^{i j}=\frac{1}{\epsilon^{2}}\left(\frac{\sqrt{|\bar{g}|}}{\sqrt{|\bar{h}|}} \bar{g}^{i j}-\bar{h}^{i j}\right)
$$

and

$$
|\bar{g}|=-\operatorname{det}\left(\bar{g}_{i j}\right) \quad \text { and } \quad|\bar{h}|=-\operatorname{det}\left(\bar{h}_{i j}\right)=a^{3} .
$$

The metric can be recovered from the $\bar{u}^{i j}$ by the formula

$$
\bar{g}^{i j}=\frac{1}{\sqrt{-|\bar{h}| \operatorname{det}\left(\hat{g}^{k l}\right)}} \hat{g}^{i j},
$$


where

$$
\hat{g}^{i j}=\bar{h}^{i j}+\epsilon^{2} \bar{u}^{i j}
$$

Substituting (2.7) into the standard formula for the Christoffel symbols gives

$$
\bar{\Gamma}_{i j}^{k}=\bar{\gamma}_{i j}^{k}+\epsilon^{2}\left(-\hat{g}_{l(i} \bar{D}_{j)} \bar{u}^{k l}+\frac{1}{2} \hat{g}^{k l} \hat{g}_{i m} \hat{g}_{j n} \bar{D}_{l} \bar{u}^{m n}-\frac{1}{4} \hat{g}^{k l} \hat{g}_{i j} \hat{g}_{m n} \bar{D}_{l} \bar{u}^{m n}+\frac{1}{2} \hat{g}_{l m} \delta_{(i}^{k} \bar{D}_{j)} \bar{u}^{l m}\right),
$$

where $\left(\hat{g}_{i j}\right)=\left(\hat{g}^{i j}\right)^{-1}$ and $\bar{D}_{k}$ is the $\bar{h}_{i j}$ covariant derivative. Using this formula, the Einstein tensor $\bar{G}^{i j}$ of the metric $\bar{g}_{i j}$ is given by

$$
|\bar{g}| \bar{G}^{i j}=\frac{\epsilon^{2}}{2}|\bar{h}|\left[\hat{g}^{k l} \bar{D}_{k} \bar{D}_{l} \bar{u}^{i j}+\epsilon^{2}\left(a_{1}^{i j}+a_{2}^{i j}+a_{3}^{i j}\right)+b^{i j}+\epsilon c_{1}^{i j}+\epsilon^{2} c_{2}^{i j}+4 \epsilon^{2} \overline{\mathcal{T}}^{i j}\right]
$$

where

$$
\begin{aligned}
& a_{1}^{i j}=\frac{1}{2}\left(\frac{1}{2} \hat{g}_{k l} \hat{g}_{m n}-\hat{g}_{k m} \hat{g}_{l n}\right)\left(\hat{g}^{i p} \hat{g}^{j q}-\frac{1}{2} \hat{g}^{i j} \hat{g}^{p q}\right) \bar{D}_{p} \bar{u}^{k l} \bar{D}_{q} \bar{u}^{m n}, \\
& a_{2}^{i j}=2 \hat{g}_{k l}\left(\hat{g}^{n(i} \bar{D}_{m} \bar{u}^{j) l} \bar{D}_{n} \bar{u}^{k m}-\frac{1}{2} \hat{g}^{i j} \bar{D}_{m} \bar{u}^{k n} \bar{D}_{n} \bar{u}^{m l}-\hat{g}^{m n} \bar{D}_{m} \bar{u}^{i k} \bar{D}_{n} \bar{u}^{j l}\right), \\
& a_{3}^{i j}=\bar{D}_{k} \bar{u}^{i j} \bar{D}_{l} \bar{u}^{k l}-\bar{D}_{k} \bar{u}^{i l} \bar{D}_{l} \bar{u}^{j k} \\
& b^{i j}=\hat{g}^{i j} \bar{D}_{k} \bar{D}_{l} \bar{u}^{k l}-2 \bar{D}_{l} \bar{D}_{k} \bar{u}^{k(i} \hat{g}^{j) l}, \\
& c_{1}^{i j}=-\left(\bar{h}^{i j} \epsilon \bar{u}^{k l}+\epsilon \bar{u}^{i j} \bar{h}^{k l}\right) \frac{1}{\epsilon^{2}} \overline{\mathcal{R}}_{k l}+\frac{2}{\epsilon^{2}} \overline{\mathcal{R}}_{l k m}{ }^{(i} \epsilon \bar{u}^{j) k} \bar{h}^{l m}+\frac{2}{\epsilon^{2}} \overline{\mathcal{R}}_{l k m}{ }^{(i} \bar{h}^{j) k} \epsilon \bar{u}^{l m}, \\
& c_{2}^{i j}=-\epsilon \bar{u}^{i j} \epsilon \bar{u}^{k l} \frac{1}{\epsilon^{2}} \overline{\mathcal{R}}_{k l}+\frac{2}{\epsilon^{2}} \overline{\mathcal{R}}_{l k m}{ }^{(i} \epsilon \bar{u}^{j) k} \epsilon \bar{u}^{l m},
\end{aligned}
$$

and

$$
\overline{\mathcal{T}}^{i j}=\mu \bar{\xi}^{i} \bar{\xi}^{j}, \quad \bar{\xi}^{i}=\frac{1}{\epsilon} \delta_{0}^{i} .
$$

For later use, we define

$$
c_{k l}^{i j}=-\frac{1}{\epsilon^{2}} \overline{\mathcal{R}}_{k l} \bar{h}^{i j}-\frac{1}{\epsilon^{2}} \overline{\mathcal{R}} \delta_{k}^{i} \delta_{l}^{j}+\frac{2}{\epsilon^{2}} \overline{\mathcal{R}}_{k}{ }^{(i} \delta_{l}^{j)}+\frac{2}{\epsilon^{2}} \overline{\mathcal{R}}_{k m l}{ }^{(i} \bar{h}^{j) m}
$$

so that

To fix the gauge, we set

$$
c_{1}^{i j}=c_{k l}^{i j} \epsilon \bar{u}^{k l} .
$$

$$
\bar{D}_{i} \bar{u}^{i j}=0 .
$$

For $\epsilon>0$, it is clear from (2.5) that this is equivalent to

$$
\bar{D}_{i}\left(\sqrt{|\bar{g}|} \bar{g}^{i j}\right)=0
$$

and this is easily seen to be equivalent to the harmonic coordinate condition

$$
\bar{g}^{i j}\left(\bar{\Gamma}_{i j}^{k}-\bar{\gamma}_{i j}^{k}\right)=0 .
$$

Defining the reduced Einstein tensor $\bar{G}_{R}^{i j}$ by

$$
\bar{G}_{R}^{i j}=\frac{1}{\epsilon^{2}} \frac{|\bar{g}|}{|\bar{h}|} \bar{G}^{i j}-b^{i j}=\frac{1}{2}\left(\hat{g}^{k l} \bar{D}_{k} \bar{D}_{l} \bar{u}^{i j}+\epsilon^{2}\left(a_{1}^{i j}+a_{2}^{i j}+a_{3}^{i j}\right)+\epsilon c_{1}^{i j}+\epsilon^{2} c_{2}^{i j}+4 \epsilon^{2} \overline{\mathcal{T}}^{i j}\right),
$$

Einstein's equations $\bar{G}^{i j}=2 \epsilon^{4} \bar{T}^{i j}$ in the gauge (2.19) become

$$
\bar{G}_{R}^{i j}=2 \epsilon^{2} \frac{|\bar{g}|}{|\bar{h}|} \bar{T}^{i j}
$$

where

$$
\bar{T}^{i j}=\left(\rho+\epsilon^{2} p\right) \bar{v}^{i} \bar{v}^{j}+p \bar{g}^{i j} \quad \text { and } \quad \bar{v}^{i} \bar{v}_{j}=-\frac{1}{\epsilon^{2}} .
$$


To write the reduced Einstein equations (2.21) in first order form, we introduce the variables

$$
u^{i j}=\epsilon \bar{u}^{i j} \quad \text { and } \quad u_{k}^{i j}=\bar{D}_{k} \bar{u}^{i j} .
$$

With these variables, we have that

$$
\bar{D}_{k} u_{l}^{i j}=\bar{D}_{l} u_{k}^{i j}-\frac{2}{\epsilon} \overline{\mathcal{R}}_{k l m}{ }^{(i} u^{j) m},
$$

or equivalently

$$
\bar{\partial}_{k} u_{l}^{i j}=\bar{\partial}_{l} u_{k}^{i j}+2 \bar{\gamma}_{l m}^{(i} u_{k}^{j) m}-2 \bar{\gamma}_{k m}^{(i} u_{l}^{j) m}-\frac{2}{\epsilon} \overline{\mathcal{R}}_{k l m}{ }^{(i} u^{j) m} .
$$

In particular, this implies that

$$
\bar{\partial}_{0} u_{I}^{i j}=\bar{\partial}_{I} u_{0}^{i j}+2 \bar{\gamma}_{I m}^{(i} u_{0}^{j) m}-2 \bar{\gamma}_{0 m}^{(i} u_{I}^{j) m}-\frac{2}{\epsilon} \overline{\mathcal{R}}_{0 I m}{ }^{(i} u^{j) m},
$$

and hence

$$
\begin{aligned}
\hat{g}^{k l} \bar{D}_{k} \bar{D}_{l} \bar{u}^{i j} & =\hat{g}^{00} \bar{\partial}_{0} u_{0}^{i j}+\hat{g}^{0 I} \bar{\partial}_{0} u_{I}^{i j}+\hat{g}^{0 I} \bar{\partial}_{I} u_{0}^{i j}+\hat{g}^{I J} \bar{\partial}_{I} u_{J}^{i j}+\hat{g}^{k l}\left(-\bar{\gamma}_{k l}^{m} u_{m}^{i j}+2 \bar{\gamma}_{k m}^{(i} u_{l}^{j) m}\right) \\
& =\hat{g}^{00} \bar{\partial}_{0} u_{0}^{i j}+2 \epsilon u^{0 I} \bar{\partial}_{I} u_{0}^{i j}+\hat{g}^{I J} \bar{\partial}_{I} u_{J}^{i j}+\epsilon d_{1}^{i j}+\epsilon^{2} d_{2}^{i j},
\end{aligned}
$$

where

$$
d_{1}^{i j}=\bar{h}^{k l}\left(-\frac{1}{\epsilon} \bar{\gamma}_{k l}^{m} u_{m}^{i j}+\frac{2}{\epsilon} \bar{\gamma}_{k m}^{(i} u_{l}^{j) m}\right)
$$

and

$$
d_{2}^{i j}=u^{k l}\left(-\frac{1}{\epsilon} \bar{\gamma}_{k l}^{m} u_{m}^{i j}+\frac{2}{\epsilon} \bar{\gamma}_{k m}^{(i} u_{l}^{j) m}\right)+u^{0 I}\left(\frac{2}{\epsilon} \bar{\gamma}_{I m}^{(i} u_{0}^{j) m}-\frac{2}{\epsilon} \bar{\gamma}_{0 m}^{(i} u_{I}^{j) m}-\frac{2}{\epsilon^{2}} \overline{\mathcal{R}}_{0 I m}{ }^{(i} u^{j) m}\right) .
$$

Using (2.1)-(2.2), we find that

$$
d_{1}^{i j}=d_{k l}^{i j m} u_{m}^{k l}
$$

where

$$
d_{k l}^{i j m}=\frac{a^{\prime}}{a}\left(-\frac{3}{2} \delta_{k}^{i} \delta_{l}^{j} \delta_{0}^{m}+\delta_{0}^{(i} \delta_{k}^{j)} \delta_{l}^{I} \delta_{I}^{m}+\frac{1}{a} \delta^{I(i} \delta_{k}^{j)} \delta_{I}^{m} \delta_{l}^{0}-\delta_{I}^{(i} \delta_{k}^{j)} \delta_{l}^{I} \delta_{0}^{m}\right)
$$

Setting

$$
e_{J k l}^{i j m}=\frac{2}{\epsilon} \bar{\gamma}_{J k}^{(i} \delta_{l}^{j)} \delta_{0}^{m}-\frac{2}{\epsilon} \bar{\gamma}_{0 k}^{(i} \delta_{l}^{j)} \delta_{J}^{m}
$$

and

$$
f_{J}^{i j}=-\frac{2}{\epsilon^{2}} \overline{\mathcal{R}}_{0 J l}{ }^{(i} \delta_{k}^{j)},
$$

equations (2.25) and (2.26) can be used to write the reduced Einstein equations (2.21) in the following first order form

$$
\begin{gathered}
-a \hat{g}^{00} \partial_{t} u_{0}^{i j}=2 a u^{0 I} \partial_{I} u_{0}^{i j}+\frac{1}{\epsilon} a \hat{g}^{I J} \partial_{I} u_{J}^{i j}+a\left(c_{k l}^{i j} u^{k l}+d_{k l}^{i j m} u_{m}^{k l}\right) \\
+\epsilon a\left(a_{1}^{i j}+a_{2}^{i j}+a_{3}^{i j}+c_{2}^{i j}+d_{2}^{i j}\right)+4 \epsilon a\left(\overline{\mathcal{T}}^{i j}-\frac{|\bar{g}|}{|\bar{h}|} \bar{T}^{i j}\right), \\
a \hat{g}^{I J} \partial_{t} u_{J}^{i j}=\frac{1}{\epsilon} a \hat{g}^{I J} \partial_{J} u_{0}^{i j}+a \hat{g}^{I J}\left(e_{J k l}^{i j m} u_{m}^{k l}+f_{J}^{i j} u^{k l}\right),
\end{gathered}
$$

and

$$
\partial_{t} u^{i j}=u_{0}^{i j}-\frac{2}{\epsilon} \bar{\gamma}_{0 k}^{(i} u^{j) k}
$$


To be correctly defined, the reduced Einstein equations (2.33)-(2.35) require that the matrix $\hat{g}^{i j}$ is invertible. By assumption, $\sigma(\epsilon)$ is analytic in a neighborhood of $\epsilon=0$ and $\sigma(0)=\sigma_{0}>0$ (see (1.23)). Thus there exists an $\epsilon_{0}>0$ such that

$$
0<\frac{\sigma_{0}}{2} \leq \sigma(\epsilon) \leq 2 \sigma_{0} \quad \forall \epsilon \in\left(-\epsilon_{0}, \epsilon_{0}\right) .
$$

For fixed $-\sqrt{4 /\left(3 \sigma_{0}\right)}<\tau_{0}<0$ and $\tau_{1}>0$, it is clear from (1.9) that

$$
\left(\sqrt{\frac{3 \mu_{0}}{4}} \tau_{0}+1\right)^{\frac{4}{3}} \leq a_{\epsilon}(t) \leq\left(\sqrt{3 \sigma_{0}} \tau_{1}+1\right)^{\frac{4}{3}}
$$

for all $(t, \epsilon) \in\left[\tau_{0}, \tau_{1}\right] \times\left(-\epsilon_{0}, \epsilon_{0}\right)$. This implies that the set

$$
\mathcal{V}=\left\{\left(r^{i j}\right) \mid \operatorname{det}\left(\bar{h}^{i j}+r^{i j}\right)>0 \forall(t, \epsilon) \in\left[\tau_{0}, \tau_{1}\right] \times\left(-\epsilon_{0}, \epsilon_{0}\right)\right\}
$$

is open and contains the origin $\left(r^{i j}\right)=0$, and moreover that the reduced Einstein equations (2.33)-(2.35) are well defined for all $t \in\left(\tau_{0}, \tau_{1}\right), \epsilon \in\left(0, \epsilon_{0}\right)$, and $\left(\epsilon u^{i j}\right) \in \mathcal{V}$. We also note that

$$
\begin{aligned}
4 \epsilon^{2}\left(\frac{|\bar{g}|}{|\bar{h}|} \bar{T}^{i j}-\overline{\mathcal{T}}^{i j}\right)= & 4(\rho-\mu) \delta_{0}^{i} \delta_{0}^{j} \\
& +\epsilon\left[8 \delta_{0}^{(i} \rho w^{j)}+4 \delta_{0}^{i} \delta_{0}^{j} \rho \bar{h}_{k l} u^{k l}\right]+\epsilon^{2} S_{0}^{i j}\left(\epsilon, \sigma, u^{k l}, \rho, w^{k}\right),
\end{aligned}
$$

where the map $S_{0}^{i j}$ is analytic in all variables provided that $(\epsilon, \sigma) \in\left(-\epsilon_{0}, \epsilon_{0}\right) \times\left(\sigma_{0} / 2,2 \sigma_{0}\right)$, and $\left(\epsilon u^{i j}\right) \in \mathcal{V}$.

2.2. Regularized Euler equations. In the coordinates $\left(\bar{x}^{i}\right)$, the Euler equations are given by

$$
\bar{\nabla}_{i} \bar{T}^{i j}=0
$$

where $\bar{T}^{i j}=\left(\rho+\epsilon^{2} p\right) \bar{v}^{i} \bar{v}^{j}+p \bar{g}^{i j}$ and the fluid velocity $\bar{v}^{i}$ is normalized according to

$$
\bar{v}_{i} \bar{v}^{i}=-\frac{1}{\epsilon^{2}} .
$$

To derive a symmetric hyperbolic system for the Euler system, we follow the method of [1] and differentiate (2.38) to get

which in turn implies

$$
\bar{v}_{i} \bar{\nabla}_{j} \bar{v}^{i}=0
$$

$$
\bar{v}^{j} \bar{v}_{i} \bar{\nabla}_{j} \bar{v}^{i}=0
$$

Writing out (2.37) explicitly, we have

$$
\left(\bar{\partial}_{i} \rho+\epsilon^{2} \bar{\partial}_{i} p\right) \bar{v}^{i} \bar{v}^{j}+\left(\rho+\epsilon^{2} p\right)\left(\bar{v}^{j} \bar{\nabla}_{i} \bar{v}^{i}+\bar{v}^{i} \bar{\nabla}_{i} \bar{v}^{j}\right)+\bar{g}^{i j} \bar{\partial}_{i} p=0 .
$$

Next, we observe that the operator

$$
L_{i}^{j}=\delta_{i}^{j}+\epsilon^{2} \bar{v}^{j} \bar{v}_{i}
$$

projects into subspace orthogonal to the fluid velocity $\bar{v}^{i}$, i.e. $L_{i}^{j} L_{k}^{i}=L_{k}^{j}$ and $L_{i}^{j} \bar{v}^{i}=0$. Applying this operator to project (2.41) into components parallel and orthogonal to $\bar{v}^{i}$ yields, after using the relations (2.38)-(2.40), the following system

$$
\begin{gathered}
\bar{v}^{i} \bar{\partial}_{i} \rho+\left(\rho+\epsilon^{2} p\right) L_{j}^{i} \bar{\nabla}_{i} \bar{v}^{j}=0, \\
M_{i j} \bar{v}^{k} \bar{\nabla}_{k} \bar{v}^{j}+\frac{1}{\rho+\epsilon^{2} p} L_{j}^{i} \bar{\partial}_{i} p=0,
\end{gathered}
$$

where

$$
M_{i j}=\bar{g}_{i j}+2 \epsilon^{2} \bar{v}_{i} \bar{v}_{j}
$$

\footnotetext{
${ }^{2}$ The bound $0<\frac{\sigma_{0}}{2} \leq \sigma(\epsilon) \leq 2 \sigma_{0} \quad \forall \epsilon \in\left(-\epsilon_{0}, \epsilon_{0}\right)$ is somewhat arbitrary and could be replaced by any bound of the form $0<\frac{\sigma_{0}}{C} \leq \sigma(\epsilon) \leq C \sigma_{0}$. However, as we are interested in the limit $\epsilon \searrow 0$, nothing is missed by assuming that $C=2$.
} 
Changing to the Makino density variable $\alpha$ (see (1.15)) and multiplying (2.42) by the square of the function

$$
f=\left(1+\frac{1}{4 n(n+1)}(\epsilon \alpha)^{2}\right)
$$

transforms the Euler equations (2.42) $-(2.43)$ into

$$
\begin{gathered}
f^{2} \bar{v}^{i} \bar{\partial}_{i} \alpha+\frac{\alpha}{2 n f} L_{j}^{i} \bar{\nabla}_{i} \bar{v}^{j}=0, \\
M_{i j} \bar{v}^{k} \bar{\nabla}_{k} \bar{v}^{j}+\frac{\alpha}{2 n f} L_{j}^{i} \bar{\partial}_{i} \alpha=0 .
\end{gathered}
$$

As discussed in the introduction, we need to introduce a new fluid four-vector by

$$
w^{i}=\bar{v}^{i}-\bar{\xi}^{i}=\bar{v}^{i}-\frac{\delta_{0}^{i}}{\epsilon} .
$$

So, letting

$$
W_{M}=\left(\alpha, w^{i}\right)^{T}
$$

allows us to write the system (2.44)-(2.45) as

$$
A_{M}^{0} \partial_{t} W_{M}=A_{M}^{I} \partial_{I} W_{M}+F_{M},
$$

where

$$
\begin{aligned}
A_{M}^{0} & =\left(\begin{array}{cc}
f^{2}\left(1+\epsilon w^{0}\right) & \frac{\epsilon \alpha}{2 n f} L_{j}^{0} \\
\frac{\epsilon \alpha}{2 n f} L_{i}^{0} & M_{i j}\left(1+\epsilon w^{0}\right)
\end{array}\right), \\
A_{M}^{I} & =\left(\begin{array}{cc}
-f^{2} w^{I} & -\frac{\alpha}{2 n f} L_{j}^{I} \\
-\frac{\alpha}{2 n f} L_{i}^{I} & -M_{i j} w^{I}
\end{array}\right)
\end{aligned}
$$

and

$$
F_{M}=\left(\begin{array}{c}
\frac{\alpha}{2 n f} L_{j}^{i}\left(\bar{\gamma}_{i l}^{j}-\bar{\Gamma}_{i l}^{j}\right) \bar{v}^{l}-\frac{\alpha}{2 n f} L_{j}^{i} \bar{\gamma}_{i l}^{j} \bar{v}^{l} \\
M_{i j}\left(\bar{\gamma}_{k l}^{j}-\bar{\Gamma}_{k l}^{j}\right) \bar{v}^{k} \bar{v}^{l}-M_{i j} \bar{\gamma}_{k l}^{j} \bar{v}^{k} \bar{v}^{l}
\end{array}\right) .
$$

Next, a straightforward calculation using (2.7) and (2.9) shows that

$$
\begin{aligned}
& M_{i j}=\bar{h}_{i j}+2 \delta_{i}^{0} \delta_{j}^{0}+\epsilon m_{i j}\left(\epsilon, \sigma, u^{k l}, w^{k}\right), \\
& L_{j}^{i}=\delta_{j}^{i}-\delta_{0}^{i} \delta_{j}^{0}+\epsilon \ell_{i}^{j}\left(\epsilon, \sigma, u^{k l}, w^{k}\right), \\
& L_{j}^{i}\left(\bar{\gamma}_{i l}^{j}-\bar{\Gamma}_{i l}^{j}\right) \bar{v}^{l}=\epsilon q_{0}\left(\epsilon, \sigma, u^{i j}, u_{k}^{i j}, w^{i}\right), \\
& L_{j}^{i} \bar{\gamma}_{i l}^{j} \bar{v}^{l}=\frac{3}{2} \frac{a^{\prime}}{a}+\epsilon q_{1}\left(\epsilon, \sigma, u^{i j}, w^{i}\right), \\
& \left(\bar{\gamma}_{k l}^{j}-\bar{\Gamma}_{k l}^{j}\right) \bar{v}^{k} \bar{v}^{l}=-u_{0}^{j 0}+\frac{1}{4} \delta^{j 0}\left(3 u_{0}^{00}-a \delta_{K L} u_{0}^{K L}\right) \\
& \quad-\frac{1}{4} \delta^{j I}\left(\frac{1}{a} u_{I}^{00}+\delta_{K L} u_{I}^{K L}\right)+\epsilon q_{0}^{j}\left(\epsilon, \sigma, u^{i j}, u_{k}^{i j}, w^{i}\right), \\
& \bar{\gamma}_{k l}^{j} \bar{v}^{k} \bar{v}^{l}=\frac{a^{\prime}}{a} \delta_{I}^{j} w^{I}+\epsilon\left[\frac{1}{\epsilon} \bar{\gamma}_{k l}^{j} w^{k} w^{l}\right],
\end{aligned}
$$


T.A. OLIYNYK

where the maps $m_{i j}, \ell_{i}^{j}, q_{0}, q_{1}$, and $q_{0}^{j}$ are analytic in all their variables provided that $(\epsilon, \sigma) \in\left(-\epsilon_{0}, \epsilon_{0}\right) \times$ $\left(\sigma_{0} / 2,2 \sigma\right)$ and $\left(\epsilon u^{i j}\right) \in \mathcal{V}$. Using these expressions, we can decompose $A_{M}^{0}, A_{M}^{I}$, and $F_{M}$ as

$$
\begin{aligned}
& A_{M}^{0}=A_{M, 0}^{0}+\epsilon A_{M, 1}^{0}\left(\epsilon, \sigma, u^{i j}, \alpha, w^{i}\right), \\
& A_{M}^{I}=A_{M, 0}^{I}+\epsilon A_{M, 1}^{I}\left(\epsilon, \sigma, u^{i j}, \alpha, w^{i}\right), \\
& F_{M}=F_{M, 0}+\epsilon F_{M, 1}\left(\epsilon, \sigma, u^{i j}, u_{k}^{i j}, \alpha, w^{i}\right),
\end{aligned}
$$

where

$$
\begin{aligned}
& A_{M, 0}^{0}=\left(\begin{array}{cc}
1 & 0 \\
0 & \bar{h}_{i j}+2 \delta_{i}^{0} \delta_{j}^{0}
\end{array}\right), \\
& A_{M, 0}^{I}=\left(\begin{array}{cc}
-w^{I} & -\frac{\alpha}{2 n} \delta_{j}^{I} \\
-\frac{\alpha}{2 n} \delta_{i}^{I} & -\left(\bar{h}_{i j}+2 \delta_{i}^{0} \delta_{j}^{0}\right) w^{I}
\end{array}\right),
\end{aligned}
$$

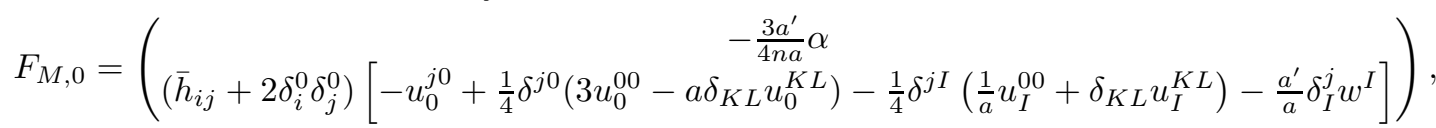

and the maps $A_{M, 1}^{0}, A_{M, 0}^{I}, F_{M, 1}$ are analytic in all their variables provided that $(\epsilon, \sigma) \in\left(-\epsilon_{0}, \epsilon_{0}\right) \times$ $\left(\sigma_{0} / 2,2 \sigma_{0}\right)$ and $\left(\epsilon u^{i j}\right) \in \mathcal{V}$.

2.3. A nonlocal symmetric hyperbolic formulation. To bring the reduced Einstein equations (2.33)-(2.35) into a form that is suitable to analyze the limit $\epsilon \searrow 0$, we replace the $u_{J}^{i j}$ with the variables

$$
W_{I}^{i j}=u_{I}^{i j}-\delta_{0}^{i} \delta_{0}^{j} \partial_{I} \Phi
$$

where $\Phi$ satisfies the Poisson equation

$$
\Delta \Phi=4 a \Pi(\rho-\mu) .
$$

where $\Delta=\delta^{I J} \partial_{I} \partial_{J}$ is the flat Laplacian. In addition to $\Phi$, we also need the time derivative

$$
\dot{\Phi}=\partial_{t} \Phi
$$

which satisfies

$$
\Delta \dot{\Phi}=4 a \Pi\left(\partial_{t}(\rho-\mu)\right)+4 a^{\prime} \Pi(\rho-\mu) .
$$

Using (2.42)-(2.43) to replace the time derivatives of $\rho$ and $\partial_{t} w^{i}$ in favor of spatial derivatives, we find that

$$
\begin{aligned}
\partial_{t}(\rho-\mu)=-\frac{3}{2} \frac{a^{\prime}}{a}(\rho-\mu) & -\partial_{I}\left(\rho w^{I}\right)+\epsilon\left[\partial_{I}\left(\rho w^{I} w^{0}\right)+\rho\left(\frac{3}{4} u_{0}^{00}-\frac{a}{4} \delta_{I J} u_{0}^{I J}\right)\right] \\
& -\epsilon^{2} \alpha^{2}\left[Q_{0}\left(\epsilon, \sigma, u^{i j}, \alpha, w^{i}\right)+Q_{1}\left(\epsilon, \sigma, u^{i j}, \alpha, w^{i}, \partial_{I} \alpha, \partial_{I} w^{i}\right)\right],
\end{aligned}
$$

where $Q_{\nu}(\nu=0,1)$ are analytic in all variables for $(\epsilon, \sigma) \in\left(-\epsilon_{0}, \epsilon_{0}\right) \times\left(\sigma_{0} / 2,2 \sigma_{0}\right)$, and $\left(\epsilon u^{i j}\right) \in \mathcal{V}$, and $Q_{1}$ is linear in $\left(\partial_{I} \alpha, \partial_{I} w^{i}\right)$.

Letting,

$$
\phi=-\frac{4}{\epsilon}\langle\hat{1} \mid \rho-\mu\rangle_{L^{2}}
$$

we see from (2.62) that $\phi$ satisfies

$$
\phi^{\prime}=-\frac{3}{2} \frac{a^{\prime}}{a} \phi-4\left[\left\langle\rho \mid \frac{3}{4} u_{0}^{00}-\frac{a}{4} \delta_{I J} u_{0}^{I J}\right\rangle_{L^{2}}\right]+4 \epsilon\left\langle\alpha^{2} \hat{1} \mid Q_{0}+Q_{1}\right\rangle_{L^{2}} .
$$

Define

$$
W=\left(u_{0}^{i j}, W_{I}^{i j}, u^{i j}, \phi^{i j}, \alpha, w^{j}\right)^{T}
$$


(2.33)-(2.35), (2.36), (2.48), (2.49)-(2.51), (2.52)-(2.54), (2.56)-(2.57), (2.58), (2.63) and (2.64) that $W$ satisfies

$$
A^{0} \partial_{t} W=\frac{1}{\epsilon} C^{I} \partial_{I} W+\left(A_{0}^{I}+\epsilon A_{1}^{I}\right) \partial_{I} W+F_{0}+\epsilon F_{1},
$$

where

$$
\begin{aligned}
& A^{0}=\left(\begin{array}{cc}
A_{G}^{0} & 0 \\
0 & A_{M, 0}^{0}+\epsilon A_{M, 1}^{0}
\end{array}\right), \\
& A_{G}^{0}=\left(\begin{array}{cccc}
a\left(1-\epsilon u^{00}\right) & 0 & 0 & 0 \\
0 & \left(\delta^{I J}+\epsilon a u^{I J}\right) & 0 & 0 \\
0 & 0 & 1 & 0 \\
0 & 0 & 0 & 1
\end{array}\right) \text {, } \\
& A_{0}^{I}=\left(\begin{array}{cc}
A_{G}^{I} & 0 \\
0 & A_{M, 0}^{I}
\end{array}\right), \\
& A_{G}^{I}=\left(\begin{array}{cccc}
2 a u^{0 I} & a u^{I J} & 0 & 0 \\
a u^{I J} & 0 & 0 & 0 \\
0 & 0 & 0 & 0 \\
0 & 0 & 0 & 0
\end{array}\right) \\
& A_{1}^{I}=\left(\begin{array}{cc}
0 & 0 \\
0 & A_{M, 1}^{I}
\end{array}\right) \text {, } \\
& C^{I}=\left(\begin{array}{cc}
C_{G}^{I} & 0 \\
0 & 0
\end{array}\right), \\
& C_{G}^{I}=\left(\begin{array}{cccc}
0 & \delta^{I J} & 0 & 0 \\
\delta^{I J} & 0 & 0 & 0 \\
0 & 0 & 0 & 0 \\
0 & 0 & 0 & 0
\end{array}\right),
\end{aligned}
$$

and

$$
\begin{aligned}
& F_{0}=\left(\begin{array}{c}
a\left(\delta_{0}^{i} \delta_{0}^{j}\left(u^{I J} \partial_{I} \partial_{J} \Phi+\phi-4 \rho \bar{h}_{k l} u^{k l}\right)-8 \delta_{0}^{(i} \rho w^{j)}+c_{k l}^{i j} u^{k l}+d_{k l}^{i j m} u_{m}^{k l}\right) \\
-\delta^{I J} \partial_{J} \dot{\Phi}_{0} \delta_{0}^{i} \delta_{0}^{j}+\delta^{I J}\left(e_{J k l}^{i j m} u_{m}^{k l}+f_{J}^{i j} u^{k l}\right) \\
u_{0}^{i j}-\frac{2}{\epsilon} \bar{\gamma}_{0 k}^{(i} u^{j) k} \\
-\frac{3}{2} \frac{a^{\prime}}{a} \phi-\left\langle\rho \mid 3 u_{0}^{00}-a \delta_{I J} u_{0}^{I J}\right\rangle_{L^{2}} \\
-\frac{3 a^{\prime}}{4 n a} \alpha \\
\left(\bar{h}_{i j}+2 \delta_{i}^{0} \delta_{j}^{0}\right)\left(-u_{0}^{j 0}+\frac{1}{4} \delta^{j 0}\left(3 u_{0}^{00}-a \delta_{K L} u_{0}^{K L}\right)-\frac{1}{4} \delta^{j I}\left(\frac{1}{a} u_{I}^{00}+\delta_{K L} u_{I}^{K L}\right)-\frac{a^{\prime}(t)}{a} \delta_{I}^{j} w^{I}\right)
\end{array}\right), \\
& \dot{\Phi}_{0}=-4 a \Pi\left(\partial_{I}\left(\rho w^{I}\right)+\frac{1}{2} \frac{a^{\prime}}{a}(\rho-\mu)\right), \\
& F_{1}=\left(\begin{array}{c}
a\left(a_{1}^{i j}+a_{2}^{i j}+a_{3}^{i j}+c_{2}^{i j}+d_{2}^{i j}-S_{0}^{i j}\right) \\
-\frac{1}{\epsilon} \delta^{I J} \partial_{J}\left(\dot{\Phi}-\dot{\Phi}_{0}\right) \delta_{0}^{i} \delta_{0}^{j}+a u^{I J}\left[\partial_{J} \dot{\Phi} \delta_{0}^{i} \delta_{0}^{j}+e_{J k l}^{i j m} u_{m}^{k l}+f_{J}^{i j} u^{k l}\right] \\
4\left\langle\alpha^{2} \hat{1} \mid Q_{0}+Q_{1}\right\rangle \\
\frac{1}{\epsilon}\left(F_{M, 0}-\tilde{F}_{M, 0}\right)+F_{M, 1}
\end{array}\right) .
\end{aligned}
$$

For $\epsilon>0$, equation (2.66) is completely equivalent to the Einstein-Euler equations in the harmonic gauge. It is this form of the Einstein-Euler equations that will be useful for analyzing the limit $\epsilon \searrow 0$. 
2.4. Well-posedness of the nonlocal system. The well-posedness of the non-local symmetric hyperbolic system (2.65) follows from the same arguments used in Section 2.4 of [10], and will not be repeated here. The well-posedness of the system (2.65) combined with its particular structure allows us to apply the local existence results of Schochet [13, 14 (see also 3, 4, ) to obtain the existence of one-parameter families of solutions to (2.65) on spacetime regions of the form $M=[0, T) \times \mathbb{T}^{3}$ where $T$ is independent of $\epsilon$, and also to identify the cosmological Poisson-Euler equations (1.20)-(1.22) as the correct limit equations satisfied by the $\epsilon \searrow 0$ limit of the solutions of (2.65). The details of this are presented in Sections 4 and 5 .

\section{Newtonian initial Data}

In order to solve the initial value problem for the Einstein equations, we must first construct initial data that satisfies the following constraint equations on the initial hypersurface defined by $t=0$ :

$$
\begin{aligned}
\left.\left(\bar{G}^{0 j}-2 \epsilon^{4} \bar{T}^{0 j}\right)\right|_{t=0} & =0, \\
\left.\bar{D}_{i} \bar{u}^{i j}\right|_{t=0} & =0
\end{aligned}
$$

and

$$
\left.\left(\bar{g}_{i j} \bar{v}^{i} \bar{v}^{j}+\frac{1}{\epsilon^{2}}\right)\right|_{t=0}=0
$$

which are the gravitational constraints, the harmonic gauge condition, and the fluid 4-velocity normalization, respectively.

To find 1-parameter families of solutions to these equations, we adapt the method developed by Lottermoser in [6. We begin by writing (3.2) as (see (2.23) $)$

$$
\left.u_{j}^{k j}\right|_{t=0}=0
$$

which shows that

$$
\left.\partial_{J} u_{k}^{k j}\right|_{t=0}=0
$$

and hence, that

$$
\left.\bar{D}_{i} u_{k}^{k j}\right|_{t=0}=\delta_{i}^{0} \epsilon \partial_{t} u_{k}^{k j}
$$

Substituting this into (2.14) yields

$$
\left.b^{0 j}\right|_{t=0}=-\hat{g}^{00} \epsilon \partial_{t} u_{k}^{j k} .
$$

Setting

$$
H^{i j}=\left.\left(\hat{g}^{k l} \bar{D}_{k} \bar{D}_{l} \bar{u}^{i j}+b^{i j}\right)\right|_{t=0},
$$

it follows directly from (2.26) and (3.7) that

$$
H^{0 j}=\hat{g}^{I J} \partial_{I} u_{J}^{0 j}-\hat{g}^{00} \epsilon \partial_{t} u_{I}^{j I}+2 \epsilon u^{0 I} \partial_{I} u_{0}^{0 j}+\epsilon d_{1}^{0 j}+\epsilon^{2} d_{2}^{0 j} .
$$

Setting $j=J$ in the above expression while using (3.4) gives

$$
H^{0 J}=\hat{g}^{K L} \partial_{K} u_{L}^{0 J}-\hat{g}^{00} \epsilon \partial_{t} u_{I}^{J I}-2 \epsilon u^{0 I} \partial_{I} u_{K}^{K J}+\epsilon d_{1}^{0 J}+\epsilon^{2} d_{2}^{0 J} .
$$

From the non-vanishing Christoffel symbols (2.1)-(2.2), we get that

$$
\begin{aligned}
& u_{0}^{00}=\epsilon \partial_{t} \bar{u}^{00}, \\
& u_{0}^{0 J}=\epsilon \partial_{t} \bar{u}^{0 J}+\frac{a^{\prime}}{2 a} u^{0 J}, \\
& u_{0}^{I J}=\epsilon \partial_{t} \bar{u}^{I J}+\frac{a^{\prime}}{a} u^{I J},
\end{aligned}
$$


and

$$
\begin{aligned}
& u_{K}^{00}=\partial_{K} \bar{u}^{00}+a^{\prime} u^{0 K}, \\
& u_{K}^{0 J}=\partial_{K} \bar{u}^{0 J}+\frac{a^{\prime}}{2} u^{K J}+\frac{a^{\prime}}{2 a} \delta_{K}^{J} u^{00}, \\
& u_{K}^{I J}=\partial_{K} \bar{u}^{I J}+\frac{a^{\prime}}{a} \delta_{K}^{(I} u^{J) 0} .
\end{aligned}
$$

Using (2.3)-(2.4), (3.4), and (3.10)-(3.15), a straightforward calculation (see (2.15) and (2.27) ) shows that

$$
\epsilon \partial_{t} u_{I}^{I J}+\epsilon\left(d_{1}^{0 J}+c^{0 J}\right)=\partial_{I}\left(\epsilon \partial_{t} \bar{u}^{I J}\right)+\epsilon\left[-\frac{3}{2}\left(\frac{a^{\prime}}{a}\right)^{2} u^{J 0}+\frac{a^{\prime}}{2 a} \partial_{I} \bar{u}^{J I}+\frac{a^{\prime}}{2 a^{2}} \partial_{J} \bar{u}^{00}\right] .
$$

Setting $j=0$ in (3.8) while using (2.25) and (3.4) gives

$$
\begin{aligned}
H^{00}=\hat{g}^{K L} \partial_{K} u_{L}^{00}-\partial_{I} u_{J}^{I J}+\epsilon\left[u^{00} \partial_{I} u_{J}^{I J}-\hat{g}^{00}\left(\frac{2}{\epsilon} \bar{\gamma}_{0 m}^{(0} u_{I}^{I) m}\right.\right. \\
\left.\left.\quad-\frac{2}{\epsilon} \bar{\gamma}_{I m}^{(0} u_{0}^{I) m}-\frac{2}{\epsilon^{2}} \overline{\mathcal{R}}_{0 I m}{ }^{(0} u^{I) m}\right)-2 u^{0 I} \partial_{I} u_{J}^{J 0}+d_{1}^{00}+\epsilon d_{2}^{00}\right]
\end{aligned}
$$

Next, we decompose the gravitational variables as follows

$$
\begin{aligned}
\bar{u}^{0 j} & =\frac{1}{\epsilon} y^{I} \delta_{I}^{j}+\breve{u}^{0 j}, \\
\bar{u}^{I J} & =\epsilon \breve{u}^{I J}, \\
\partial_{t} \bar{u}^{I J} & =\breve{u}_{0}^{I J},
\end{aligned}
$$

where we assume that the $y^{J}$ are constants and

$$
\int_{\mathbb{T}^{3}} \breve{u}^{0 j} d^{3} x=0 .
$$

In terms of these variables, the harmonic conditions become

$$
\begin{aligned}
\partial_{t} \bar{u}^{00} & =-\frac{1}{\epsilon} \partial_{I} \breve{u}^{I 0}-\frac{3}{2} \ln (a)^{\prime} \breve{u}^{00}-\frac{1}{2} a^{\prime} \delta_{I J} \epsilon \breve{u}^{I J}, \\
\partial_{t} \bar{u}^{0 J} & =-\partial_{I} \breve{u}^{I J}-\frac{5}{2} \ln (a)^{\prime}\left(\frac{1}{\epsilon} y^{J}+\breve{u}^{0 J}\right) .
\end{aligned}
$$

Using (2.10), (3.9), (3.16), (3.17), (3.22), and (3.23), the gravitational constraints (3.1) take the form

$$
\begin{gathered}
\Delta \breve{u}^{00}-4 a(\rho-\mu)+\epsilon\left[f_{1}^{0}(\epsilon, \sigma, y, \breve{u}, \rho, w)+f_{1}^{0}\left(\epsilon, \sigma, y, \breve{u}, \partial_{x}^{2} \breve{u}, \partial_{x} \breve{u}, \breve{u}_{0}\right)+f_{2}^{0}\left(\epsilon, \sigma, y, \breve{u}, \partial_{x} \breve{u}, \breve{u}_{0}\right)\right]=0, \\
\Delta \breve{u}^{0 J}+\epsilon a\left[\frac{a^{\prime}}{a} \partial^{J} \breve{u}^{00}+\partial_{I} \breve{u}_{0}^{I J}+y^{K L} \partial_{K} \partial_{L} \breve{u}^{0 J}\right]+\epsilon a\left[-\frac{3}{2}\left(\frac{a^{\prime}}{a}\right)^{2} y^{J}-4 \rho w^{J}\right] \\
\quad+\epsilon^{2}\left[f_{1}^{J}(\epsilon, \sigma, y, \breve{u}, \rho, w)+f_{1}^{J}\left(\epsilon, \sigma, y, \breve{u}, \partial_{x}^{2} \breve{u}, \partial_{x} \breve{u}, \breve{u}_{0}\right)+f_{2}^{J}\left(\epsilon, \sigma, y, \breve{u}, \partial_{x} \breve{u}, \breve{u}_{0}\right)\right]=0
\end{gathered}
$$

where (i) for any $R>0$ there exists an $\epsilon_{0}>0$ such that the maps $f_{\alpha}(\alpha=2,3,4)$ are analytic in all their variables provided $|\epsilon|<\epsilon_{0}, \sigma \in\left(\sigma_{0} / 2,2 \sigma_{0}\right)$ and $\left|\bar{u}^{i j}\right|<R$, (ii) $f_{2}^{j}$ is linear in $\left(\partial_{K} \partial_{L} \bar{u}^{i j}, \partial_{K} \bar{u}^{i j}, \partial_{t} \bar{u}^{i j}\right)$, and (iii) $f_{2}^{j}$ is quadratic in $\left(\partial_{t} \bar{u}^{i j}, \partial_{K} \bar{u}^{i j}\right)$. Here, $a, a^{\prime}$, and $\mu$ are determined by evaluating the formulas (1.6), (1.9) and (1.10) at $t=0$, that is

$$
a=1, \quad \mu=\sigma, \quad \text { and } \quad a^{\prime}=\sqrt{\frac{8}{3} \sigma}
$$

Also, a calculation using (2.7) shows that (3.3) can be written as

$$
w^{0}-\epsilon f_{0}\left(\epsilon, \sigma, y, \breve{u}^{i j}, w^{I}\right)=0,
$$


where the map $f_{0}$ is analytic provided $|\epsilon|<\epsilon_{0}, \sigma \in\left(\sigma_{0} / 2,2 \sigma_{0}\right),\left|\bar{u}^{i j}\right|<R$, and $\left|w^{I}\right|<R$.

Theorem 3.1. Suppose $s>3 / 2, R>0, \alpha \in H^{s}, w^{I} \in H^{s}\left(\mathbb{R}^{3}\right), \breve{u}_{0}^{I J} \in \bar{H}^{s}\left(\mathbb{S}_{3}\right), \breve{u}^{I J} \in B_{R}\left(\bar{H}^{s+1}\left(\mathbb{S}_{3}\right)\right)$. Then there exists an $\epsilon_{0}>0$, and analytic maps

$$
\begin{aligned}
& \left(-\epsilon_{0}, \epsilon_{0}\right) \ni \epsilon \longmapsto \sigma(\epsilon) \in \mathbb{R}_{>0}, \\
& \left(-\epsilon_{0}, \epsilon_{0}\right) \ni \epsilon \longmapsto w_{\epsilon}^{0} \in H^{s}, \\
& \left(-\epsilon_{0}, \epsilon_{0}\right) \ni \epsilon \longmapsto y^{J}(\epsilon) \in \mathbb{R}^{3},
\end{aligned}
$$

and

$$
\left(-\epsilon_{0}, \epsilon_{0}\right) \ni \epsilon \longmapsto \breve{u}_{\epsilon}^{0 j}=\breve{u}_{\epsilon}^{j 0} \in \bar{H}^{s}\left(\mathbb{R}^{4}\right)
$$

such that for every $\epsilon \in\left(-\epsilon_{0}, \epsilon_{0}\right)$

$$
\begin{aligned}
\left.\alpha\right|_{t=0} & =\alpha, \\
\left.\left(w_{\epsilon}^{j}\right)\right|_{t=0} & =\left(w_{\epsilon}^{0}, w^{I}\right), \\
\left.\left(\bar{u}_{\epsilon}^{i j}\right)\right|_{t=0} & =\left(\begin{array}{cc}
\breve{u}_{\epsilon}^{00} & \frac{1}{\epsilon} y^{J}(\epsilon)+\bar{u}_{\epsilon}^{0 J} \\
\frac{1}{\epsilon} y^{J}(\epsilon)+\breve{u}_{\epsilon}^{J 0} & \epsilon \breve{u}^{I J}
\end{array}\right)
\end{aligned}
$$

and

$$
\left.\left(\partial_{t} \bar{u}_{\epsilon}^{i j}\right)\right|_{t=0}=\left(\begin{array}{cc}
\left.\partial_{t} \bar{u}_{\epsilon}^{00}\right|_{t=0} & \left.\partial_{t} \bar{u}_{\epsilon}^{0 J}\right|_{t=0} \\
\left.\partial_{t} \bar{u}_{\epsilon}^{0 J}\right|_{t=0} & \breve{u}_{0}^{I J}
\end{array}\right)
$$

where

and

$$
\left.\partial_{t} \bar{u}_{\epsilon}^{00}\right|_{t=0}=-\frac{1}{\epsilon} \partial_{I} \breve{u}_{\epsilon}^{I 0}-\frac{3}{2} \ln (a)^{\prime} \breve{u}_{\epsilon}^{00}-\frac{1}{2} a^{\prime} \delta_{I J} \epsilon \breve{u}^{I J},
$$

$$
\left.\partial_{t} \bar{u}_{\epsilon}^{0 J}\right|_{t=0}=-\partial_{I} \breve{u}^{I J}-\frac{5}{2} \ln (a)^{\prime}\left(\frac{1}{\epsilon} y^{J}(\epsilon)+\breve{u}_{\epsilon}^{0 J}\right),
$$

satisfy the gravitational constraint equations (3.1), the harmonic gauge condition (3.2), and the fluid velocity normalization (3.3). Moreover, the maps $\sigma(\epsilon), w_{\epsilon}^{0}, y^{J}(\epsilon)$ and $\breve{u}_{\epsilon}^{0 j}$ admit the expansions

$$
\begin{gathered}
\sigma(\epsilon)=\sigma_{0}+\mathrm{O}(\epsilon), \quad w_{\epsilon}^{0}=\mathrm{O}(\epsilon), \quad y^{J}(\epsilon)=y_{0}^{J}+\mathrm{O}(\epsilon), \\
\breve{u}_{\epsilon}^{00}=\phi+\mathrm{O}(\epsilon), \quad \text { and } \quad \breve{u}_{\epsilon}^{0 J}=\mathrm{O}(\epsilon),
\end{gathered}
$$

where

$$
\sigma_{0}=\int_{\mathbb{T}^{3}} \rho d^{3} x, \quad y_{0}^{J}=-\frac{1}{\sigma_{0}} \int_{\mathbb{T}^{3}} \rho w^{J} d^{3} x
$$

and

$$
\phi=\Delta^{-1}\left(\rho-\sigma_{0}\right) .
$$

Proof. Applying the projection operators $\Pi$ and $\mathbb{I}-\Pi$ to the equations (3.24)-(3.25), while observing the averaging conditions (3.21) and the definitions (3.26), yields the equations

$$
\begin{aligned}
& \sigma-\langle 1 \mid \rho\rangle_{L^{2}}+\frac{\epsilon}{4}\left\langle 1 \mid F^{0}\left(\epsilon, \sigma, y, \breve{u}, \breve{u}_{0}, \alpha, w\right)\right\rangle_{L^{2}}=0, \\
& y^{J}+\frac{1}{\sigma}\left\langle 1 \mid \rho w^{J}\right\rangle_{L^{2}}-\frac{1}{4 \sigma} \epsilon\left\langle 1 \mid F^{J}\left(\epsilon, \sigma, y, \breve{u}, \breve{u}_{0}, \alpha, w\right)\right\rangle_{L^{2}}=0, \\
& \Delta \breve{u}^{00}-4 \Pi(\rho)+\epsilon \Pi\left(F^{0}\left(\epsilon, \sigma, y, \breve{u}, \breve{u}_{0}, \alpha, w\right)\right)=0,
\end{aligned}
$$


and

$$
\Delta \breve{u}^{0 J}+\epsilon \Pi\left(-4 \rho w^{J}+\epsilon F^{J}\left(\epsilon, \sigma, y, \breve{u}, \breve{u}_{0}, \alpha, w\right)\right)=0 .
$$

These equations together with (3.27) form the complete set of constraint equations to be solved

Using the same arguments as in Section 3 of [10], it can be shown that for any $s>3 / 2, R>0$, and $\sigma_{0}>0$ that there exists an $\epsilon>0$ such that the maps

$$
\begin{aligned}
\left(\epsilon, \sigma, y^{J}, \breve{u}^{i j}, \breve{u}_{0}^{I J}, \alpha, w^{j}\right) & \ni\left(-\epsilon_{0}, \epsilon_{0}\right) \times\left(\sigma_{0} / 2,2 \sigma_{0}\right) \times \mathbb{R}^{3} \times \\
& B_{R}\left(\bar{H}^{s+1}\left(\mathbb{S}_{4}\right)\right) \times H^{s}\left(\mathbb{S}_{3}\right) \times H^{s} \times H^{s}\left(\mathbb{R}^{4}\right) \mapsto F^{j} \in H^{s-1}\left(\mathbb{R}^{4}\right)
\end{aligned}
$$

and

$$
\left(\epsilon, \sigma, y, \breve{u}^{i j}, w^{I}\right) \ni\left(-\epsilon_{0}, \epsilon_{0}\right) \times\left(\sigma_{0} / 2,2 \sigma_{0}\right) \times \mathbb{R}^{3} \times B_{R}\left(\bar{H}^{s+1}\left(\mathbb{S}_{4}\right)\right) \times H^{s}\left(\mathbb{R}^{3}\right) \mapsto f_{0} \in H^{s}
$$

are analytic. Setting

$$
\psi=\left(\breve{u}^{I J}, \breve{u}_{0}^{I J}, \alpha, w^{J}\right)^{T} \quad \text { and } \quad \eta=\left(\sigma, y^{J}, \breve{u}^{0 j}, w^{0}\right),
$$

allows us to write the constraint equations (3.27), (3.28)-(3.31) as the following single equation:

$$
L(\psi, \eta)+\epsilon M(\epsilon, \psi, \eta)=0
$$

where

$$
L(\psi, \eta)=\left(\begin{array}{c}
\sigma-\langle 1 \mid \rho\rangle_{L^{2}} \\
y^{J}+\frac{1}{\sigma}\left\langle 1 \mid \rho w^{J}\right\rangle_{L^{2}} \\
\Delta \breve{u}^{0 j}-4 \Pi(\rho) \delta^{0 j} \\
w^{0}
\end{array}\right)
$$

and $M(\epsilon, \psi, \eta)$ are analytic maps. It is clear that

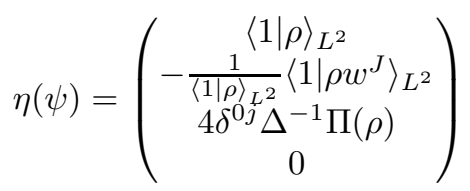

solves

$$
L(\psi, \eta(\psi))=0 .
$$

Also, a straightforward calculation shows that

$$
D_{2} L(\psi, \eta(\psi)) \cdot \delta \eta=\left(\begin{array}{c}
\delta \sigma \\
\delta y^{J}-\frac{\left\langle 1 \mid \rho w^{J}\right\rangle_{L^{2}}}{\langle 1 \mid \rho\rangle_{L^{2}}^{2}} \delta \sigma \\
\Delta \delta \breve{u}^{0 j} \\
\delta w^{0}
\end{array}\right),
$$

and it follows easily from this equation and the invertibility of the Laplacian $\Delta: \bar{H}^{s+1} \longrightarrow \bar{H}^{s-1}$ that the linear map

$$
D_{2} L(\psi, \eta(\psi)): \mathbb{R} \times \mathbb{R}^{3} \times \bar{H}^{s+1}\left(\mathbb{R}^{4}\right) \times H^{s} \longrightarrow \mathbb{R} \times \mathbb{R}^{3} \times \bar{H}^{s-1}\left(\mathbb{R}^{4}\right) \times H^{s}
$$

is an isomorphism. The proof of the Theorem now follows from (3.32)-(3.37) and the analytic version of the Implicit Function Theorem (see 2], Theorem 15.3). 


\section{LIMIT EQUATIONS}

The evolution equations (2.65) are now in a form to which the theory of singular symmetric hyperbolic equations [4,13,14] pioneered and developed by Kreiss, Klainerman, Majda, and Schochet applies. We know from the theory developed in these works that the appropriate limit equation that is satisfied by solutions of (2.65) in the limit $\epsilon \searrow 0$ is

$$
\begin{aligned}
\tilde{A}_{0}^{0} \partial_{t} W & =\tilde{A}_{0}^{I} \partial_{I} W+\tilde{F}_{0}+C^{I} \partial_{I} \omega, \\
C^{I} \partial_{I} W & =0,
\end{aligned}
$$

where

$$
\begin{aligned}
\tilde{F}_{0} & =\left.F_{0}\right|_{\epsilon=0}, \\
\tilde{A}_{0}^{0} & =\left(\begin{array}{cc}
\tilde{A}_{G, 0}^{0} & 0 \\
0 & \left.A_{0, M}\right|_{\epsilon=0}
\end{array}\right),
\end{aligned}
$$

and

$$
\tilde{A}_{G, 0}^{0}=\left(\begin{array}{cccc}
\tilde{a} & 0 & 0 & 0 \\
0 & \delta^{I J} & 0 & 0 \\
0 & 0 & 1 & 0 \\
0 & 0 & 0 & 1
\end{array}\right)
$$

As we shall shortly see, solutions to the cosmological Poisson-Euler-Makino equations (1.16)-(1.18) determine solutions to the limit equations (4.1)-(4.2). But first, we record the following local existence and uniqueness result for the Poisson-Euler-Makino equations.

Proposition 4.1. Suppose $s \geq 3+\ell, \tilde{\alpha}_{0} \in H^{k}$, and $\tilde{w}_{0}^{I} \in H^{k}\left(\mathbb{R}^{3}\right)$. Then there exists a maximal time $T_{0}$ and a unique solution

$$
\tilde{\alpha} \in X_{T_{0}, \ell, s}, \quad \tilde{w}^{I} \in X_{T_{0}, \ell, s}\left(\mathbb{R}^{3}\right), \quad \tilde{\Phi} \in X_{T_{0}, \ell+2, s+2}
$$

to the Poisson-Euler-Makino equations (1.16)-(1.18) with initial data $\left.\tilde{\alpha}\right|_{t=0}=\tilde{\alpha}_{0}$ and $\left.\tilde{w}^{I}\right|_{t=0}=\tilde{w}_{0}^{I}$.

Proof. Since the Poisson-Euler-Makino equations (1.16)-(1.18) form a (non-local) symmetric hyperbolic system, the proof follows from standard theory. For example, see [15], Chapter 16. 
Proposition 4.2. Suppose $\left\{\tilde{\alpha}, \tilde{w}^{I}, \tilde{\Phi}\right\}$ is the solution to the Poisson-Euler-Makino equations from Proposition 4.1, and let

$$
\begin{aligned}
\tilde{a} & =\exp \left(\int_{0}^{t}\left(\frac{8}{3} \int_{\mathbb{T}^{3}} \tilde{\rho}(s) d^{3} x\right)^{\frac{1}{2}} d s\right), \\
\tilde{\mu} & =\int_{\mathbb{T}^{3}} \tilde{\rho} d^{3} x \\
\zeta^{J} & =\int_{\mathbb{T}^{3}} \tilde{\rho} \tilde{w}^{J} d^{3} x \\
\tilde{u}_{0}^{i j} & =2 \delta_{0}^{(i} \delta_{J}^{j)}\left(-\left(\frac{1}{\tilde{\mu}} \zeta^{J}\right)^{\prime}-\frac{\tilde{a}^{\prime}}{2 \tilde{a}} \frac{1}{\tilde{\mu}} \zeta^{J}\right), \\
\tilde{u}^{i j} & =2 \delta_{0}^{(i} \delta_{J}^{j)}\left(-\frac{1}{\tilde{\mu}} \zeta^{J}\right), \\
\widetilde{W}_{I}^{00} & =\tilde{a}^{\prime} \tilde{u}^{0 I} \\
\widetilde{W}_{I}^{J 0} & =0 \\
\widetilde{W}_{I}^{J K} & =\frac{\tilde{a}^{\prime}}{\tilde{a}} \delta_{I}^{(J} \tilde{u}^{K) 0} \\
\tilde{\phi} & =0 \\
\tilde{w}^{i} & =\delta_{J}^{i} \tilde{w}^{J}, \\
\tilde{h}_{i j} & =-\delta_{i}^{0} \delta_{j}^{0}+\tilde{a} \delta_{i}^{I} \delta_{i}^{J} \delta_{I J}, \\
\tilde{d}_{k l}^{i j m} & =\frac{\tilde{a}^{\prime}}{\tilde{a}}\left(-\frac{3}{2} \delta_{k}^{i} \delta_{l}^{j} \delta_{0}^{m}+\delta_{0}^{(i} \delta_{k}^{j)} \delta_{l}^{I} \delta_{I}^{m}+\frac{1}{\tilde{a}} \delta^{I(i} \delta_{k}^{j)} \delta_{I}^{m} \delta_{l}^{0}-\delta_{I}^{(i} \delta_{k}^{j)} \delta_{l}^{I} \delta_{0}^{m}\right), \\
\omega_{0}^{i j} & =\delta_{0}^{i} \delta_{0}^{j} \partial_{t} \tilde{\Phi},
\end{aligned}
$$

and

$$
\omega_{I}^{i j}=\Delta^{-1}\left(-\delta_{0}^{i} \delta_{0}^{j} \delta_{I} \delta_{J} \tilde{\Phi}-\tilde{d}_{00}^{i j I} \partial_{I} \tilde{\Phi}+8 \delta_{0}^{(i} \Pi\left(\tilde{\rho} \tilde{w}^{j)}\right)+\tilde{h}_{k l} \tilde{u}^{k l} \Pi(\tilde{\rho})\right)
$$

Then

$$
\begin{aligned}
\widetilde{W} & =\left(\tilde{u}_{0}^{i j}, \widetilde{W}_{I}^{i j}, \tilde{u}^{i j}, \tilde{\phi}, \tilde{\alpha}, \tilde{w}^{i}\right)^{T}, \\
\omega & =\left(\omega_{0}^{i j}, \omega_{I}^{i j}, 0,0,0,0\right)^{T},
\end{aligned}
$$

defines a solution to the limit equations (4.1)-(4.2) on the spacetime region $M_{0}=\left[0, T_{0}\right) \times \mathbb{T}^{3}$.

Proof. The proof follows by a straightforward calculation that verifies $\widetilde{W}$ is a solution to the limit equation (4.1)-(4.2).

\section{The Newtonian Limit}

We are now ready to prove Theorem 1.1 . 
Proof of Theorem 1.1, Given $\tilde{\alpha}_{0} \in H^{s}, \tilde{w}_{0}^{I} \in H^{s}\left(\mathbb{R}^{3}\right), \breve{u}_{0}^{I J} \in \bar{H}^{s}\left(\mathbb{S}_{3}\right), \breve{u}^{I J} \in \bar{H}^{s+1}\left(\mathbb{S}_{3}\right)$, we let

$$
\begin{aligned}
\left.\alpha_{\epsilon}\right|_{t=0} & =\tilde{\alpha}_{0}, \\
\left.\left(w_{\epsilon}^{j}\right)\right|_{t=0} & =\left(w_{\epsilon}^{0}, \tilde{w}_{0}^{I}\right), \\
\left.\left(\bar{u}_{\epsilon}^{i j}\right)\right|_{t=0} & =\left(\begin{array}{cc}
\breve{u}_{\epsilon}^{00} & \frac{1}{\epsilon} y^{J}(\epsilon)+\bar{u}_{\epsilon}^{0 J} \\
\frac{1}{\epsilon} y^{J}(\epsilon)+\breve{u}_{\epsilon}^{J 0} & \epsilon \breve{u}^{I J}
\end{array}\right), \\
\left.\left(\partial_{t} \bar{u}_{\epsilon}^{i j}\right)\right|_{t=0} & =\left(\begin{array}{cc}
\left.\partial_{t} \bar{u}_{\epsilon}^{00}\right|_{t=0} & \left.\partial_{t} \bar{u}_{\epsilon}^{0 J}\right|_{t=0} \\
\left.\partial_{t} \bar{u}_{\epsilon}^{0 J}\right|_{t=0} & \breve{u}_{0}^{I J}
\end{array}\right),
\end{aligned}
$$

where

$$
\begin{aligned}
\left.\partial_{t} \bar{u}_{\epsilon}^{00}\right|_{t=0} & =-\frac{1}{\epsilon} \partial_{I} \breve{u}_{\epsilon}^{I 0}-\frac{3}{2} \ln (a)^{\prime} \breve{u}_{\epsilon}^{00}-\frac{1}{2} a^{\prime} \delta_{I J} \epsilon \breve{u}^{I J}, \\
\left.\partial_{t} \bar{u}_{\epsilon}^{0 J}\right|_{t=0} & =-\partial_{I} \breve{u}^{I J}-\frac{5}{2} \ln (a)^{\prime}\left(\frac{1}{\epsilon} y^{J}(\epsilon)+\breve{u}_{\epsilon}^{0 J}\right),
\end{aligned}
$$

be the initial data from Theorem 3.1 By construction, this data solves the constraint equations (3.1)(3.3), and depends analytically on $\epsilon$, and satisfies

$$
\begin{gathered}
\sigma_{\epsilon}=\sigma_{0}+\mathrm{O}(\epsilon), \quad w_{\epsilon}^{0}=\mathrm{O}(\epsilon), \quad y^{J}(\epsilon)=y_{0}^{J}+\mathrm{O}(\epsilon), \\
\breve{u}_{\epsilon}^{00}=\phi+\mathrm{O}(\epsilon), \quad \text { and } \quad \breve{u}_{\epsilon}^{0 J}=\mathrm{O}(\epsilon),
\end{gathered}
$$

where

$$
\sigma_{0}=\int_{\mathbb{T}^{3}} \rho d^{3} x, \quad y_{0}^{J}=-\frac{1}{\sigma_{0}} \int_{\mathbb{T}^{3}} \rho w^{J} d^{3} x
$$

and

$$
\phi=\Delta^{-1}\left(\rho-\sigma_{0}\right)
$$

A straightforward calculation then shows that

$$
W_{\epsilon}(t)=\left(u_{0, \epsilon}^{i j}(t), W_{I, \epsilon}^{i j}(t), u_{\epsilon}^{i j}(t), \phi_{\epsilon}(t), \alpha_{\epsilon}(t), w_{\epsilon}^{j}(t)\right)^{T}
$$

satisfies

$$
\left.W_{0}\right|_{t=0}=\left(\left.\tilde{u}_{0}^{i j}\right|_{t=0}, 0,\left.\tilde{u}^{i j}\right|_{t=0}, 0, \tilde{\alpha}_{0}, \delta_{I}^{j} \tilde{w}_{0}^{I}\right)
$$

where

$$
\begin{aligned}
\tilde{\rho} & =(4 K n(n+1))^{-n} \tilde{\alpha}^{2 n}, \\
\tilde{a} & =\exp \left(\int_{0}^{t}\left(\frac{8}{3} \int_{\mathbb{T}^{3}} \tilde{\rho}(s) d^{3} x\right)^{\frac{1}{2}} d s\right), \\
\tilde{\mu} & =\int_{\mathbb{T}^{3}} \tilde{\rho} d^{3} x, \\
\zeta^{J} & =\int_{\mathbb{T}^{3}} \tilde{\rho} \tilde{w}^{J} d^{3} x, \\
\tilde{u}_{0}^{i j} & =2 \delta_{0}^{(i} \delta_{J}^{j)}\left(-\left(\frac{1}{\tilde{\mu}} \zeta^{J}\right)^{\prime}-\frac{\tilde{a}^{\prime}}{2 \tilde{a}} \frac{1}{\tilde{\mu}} \zeta^{J}\right) \\
\tilde{u}^{i j} & =2 \delta_{0}^{(i} \delta_{J}^{j)}\left(-\frac{2}{\tilde{\mu}} \zeta^{J}\right),
\end{aligned}
$$


and $\left\{\tilde{\alpha}, \tilde{w}^{I}, \tilde{\Phi}\right\}$ is the solution to the Cosmological Poisson-Euler equations from Proposition 4.1 that is generated from the initial data $\left.\tilde{\alpha}\right|_{t=0}=\tilde{\alpha}_{0}$ and $\left.\tilde{w}^{I}\right|_{t=0}=\tilde{w}_{0}^{I}$. Also, it can be verified using the evolution equations (2.65) that our choice of initial data guarantees that

$$
\left\|\left.\partial_{t} W_{\epsilon}\right|_{t=0}\right\| \|_{\mathcal{H}^{s}} \lesssim 1 \quad 0<\epsilon<\epsilon_{0}
$$

where

$$
\mathcal{H}^{s}=\left(H^{s}\left(\mathbb{S}_{4}\right)\right)^{3} \times \mathbb{R} \times H^{s} \times H^{s}\left(\mathbb{R}^{4}\right) .
$$

Together, Proposition 4.2 and (5.2)-(5.3) allow us to apply Theorem 1 of [14] (see also the remarks in Section 1 of [14]) and conclude (shrinking $\epsilon_{0}$ if necessary) that for any $T<T_{0}$, there exists maps

$$
W_{\epsilon} \in \bigcap_{p=0}^{\ell+3} C^{p}\left([0, T), \mathcal{H}^{s-p}\right) \quad 0<\epsilon<\epsilon_{0},
$$

such that

(i) $W_{\epsilon}\left(t, x^{I}\right)$ solves equation (2.66) on the spacetime region $\left(t=x^{0}, x^{I}\right) \in D=[0, T) \times \mathbb{T}^{3}$,

(ii) $W_{\epsilon}$ satisfy the estimates

$$
\left\|W_{\epsilon}(t)\right\|_{\mathcal{H}^{s}}+\left\|\partial_{t} W_{\epsilon}(t)\right\|_{\mathcal{H}^{s-1}} \lesssim 1,
$$

(iv)

for all $(t, \epsilon) \in[0, T) \times\left(0, \epsilon_{0}\right)$, and

$$
\left\|W_{\epsilon}(t)-\widetilde{W}(t)\right\|_{H^{s-1}} \lesssim \epsilon
$$

for all $(t, \epsilon) \in[0, T) \times\left(0, \epsilon_{0}\right)$, where $\widetilde{W}$ is the solution of the limit equations from Proposition 4.2 .

Finally, we observe that similar arguments used in the proof of Proposition 6.1 in $[8]$ show that

$$
\left\{\bar{u}_{\epsilon}^{i j}=\epsilon^{-1} u_{\epsilon}^{i j}, \alpha_{\epsilon}, w_{\epsilon}^{i}\right\}
$$

determines, via formulas (1.15) and (1.11)-(1.13), a solution to the Einstein-Euler equations (1.1) in the harmonic gauge (2.19), and moreover, that

$$
\begin{aligned}
\epsilon \partial_{t} \bar{u}_{\epsilon}^{00} & =u_{0, \epsilon}^{00}, \\
\epsilon \partial_{t} \bar{u}^{0 J} & =u_{0, \epsilon}^{0 J}-\frac{a^{\prime}}{2 a} \epsilon \bar{u}_{\epsilon}^{0 J}, \\
\epsilon \partial_{t} \bar{u}^{I J} & =u_{0, \epsilon}^{I J}-\frac{a^{\prime}}{a} \epsilon \bar{u}_{\epsilon}^{I J},
\end{aligned}
$$

and

$$
\begin{aligned}
& \partial_{K} \bar{u}_{\epsilon}^{00}=W_{K, \epsilon}^{00}-a^{\prime} \epsilon \bar{u}_{\epsilon}^{0 K}+\partial_{K} \Phi_{\epsilon}, \\
& \partial_{K} \bar{u}_{\epsilon}^{0 J}=W_{K, \epsilon}^{0 J}-\frac{a^{\prime}}{2} \epsilon \bar{u}_{\epsilon}^{K J}+\frac{a^{\prime}}{2 a} \delta_{K}^{J} \epsilon \bar{u}_{\epsilon}^{00}, \\
& \partial_{K} \bar{u}_{\epsilon}^{I J}=W_{K, \epsilon}^{I J}-\frac{a^{\prime}}{a} \epsilon \delta_{K}^{(I} \bar{u}_{\epsilon}^{J) 0} .
\end{aligned}
$$

This combined with the statements (i)-(iii) above completes the proof. 


\section{REFERENCES}

[1] U. Brauer and L. Karp, Local existence of classical solutions of the system using weighted sobolev spaces of fractional order, Les Comptes l'Académie des sciences / Série Mathematique 345 (2007), 49-54.

[2] K. Deimling, Nonlinear functional analysis, Springer-Verlag, Berlin, 1998.

[3] S. Klainerman and A. Majda, Compressible and incompressible fluids, Comm. Pure Appl. Math. 35 (1982), 629-651.

[4] H.O. Kreiss, Problems with different time scales for partial differential equations, Comm. Pure Appl. Math. 33 (1980), 399-439.

[5] H.P. Künzle Covariant Newtonian limit of Lorentz space-times, Gen. Rel. Grav. 7 (1976), 445-457.

[6] M. Lottermoser, A convergent post-Newtonian approximation for the constraints in general relativity, Ann. Inst. Henri Poincaré 57 (1992), 279-317.

[7] T. Makino, "On a local existence theorem for the evolution equation of gaseous stars", in Patterns and Waves, edited by T. Nishida, M. Mimura, and H. Fujii, North-Holland, Amsterdam, 1986.

[8] T.A. Oliynyk, The Newtonian limit for perfect fluids, Commun. Math. Phys. 276 (2007), 131-188.

[9] T.A. Oliynyk, Post-Newtonian expansions for perfect fluids, Commun. Math. Phys. 288 (2009), 847-886.

[10] T.A. Oliynyk, Cosmological post-Newtonian expansions to arbitrary order, Commun. Math. Phys. (accepted) [preprint: arXiv:0908.2836].

[11] A.D. Rendall, The initial value problem for a class of general relativistic fluid bodies, J. Math. Phys. 33 (1992), 1047-1053.

[12] C. Rüede and N. Straumann, On Newton-Cartan cosmology, Helv.Phys.Acta 70 (1997), 318-335.

[13] S. Schochet, Symmetric hyperbolic systems with a large parameter, Comm. partial differential equations, 11 (1986), 1627-1651.

[14] S. Schochet, Asymptotics for symmetric hyperbolic systems with a large parameter, J. differential equations 75 (1988), $1-27$.

[15] M.E Taylor, Partial differential equations III, nonlinear equations, Springer, New York, 1996.

School of Mathematical Sciences, Monash University, ViC 3800, Australia

E-mail address: todd.oliynyk@sci.monash.edu.au 